\title{
CAPACIDAD DE UNA MEDIDA DE INFLACIÓN PERCIBIDA PARA PREDECIR EL ÍNDICE DE CONFIANZA DEL CONSUMIDOR, LAS EXPECTATIVAS DE INFLACIÓN Y LA INFLACIÓN EN COSTA RICA, 2006-2012
}

\author{
Diego Agüero-Morera ${ }^{1}$ \\ Andrés Bien-Briceño ${ }^{2}$ \\ Mauricio Villalobos-Delgado ${ }^{3}$
}

Aprobado: 16/11/2016

\section{RESUMEN}

En este estudio se construyó el Índice de Precios Percibidos al Comprador (IPPC) para estimar la inflación percibida en Costa Rica de julio 2006 a diciembre 2012. Con base en los fundamentos teóricos de Brachinger (2006,2008), se modificó la metodología del índice de Precios del Consumidor (IPC), dándole un mayor peso a los bienes de la canasta que se compran con más frecuencia. Se utilizó el IPPC para estimar modelos ARMA y ARDL, donde se evidenció una buena capacidad de pronóstico para el IPC de estratos bajos y las expectativas de inflación. Al comparar el IPC y el IPPC, la diferencia relativa entre los dos índices tiende a ser superior para niveles más altos de inflación, siendo mayor el índice propuesto. Además, artículos comprados semanalmente como la gasolina, el casado y el transporte en taxi predominan en la media de la variación interanual mensual del IPPC; mientras que el peso de bienes comprados semestral o anualmente es prácticamente nulo.

PALABRAS CLAVE: PERCEPCIÓN, FRECUENCIA DE COMPRA, POLÍTICA MONETARIA, BANCO CENTRAL.

\section{ABSTRACT}

In this study we computed the IPPC (Buyer's Perceived Inflation Index) in order to approximate the perceptions of consumer price inflation in Costa Rica from July 2006 to December 2012. Based on Brachinger's theory, the CPI methodology was modified by giving greater weight to items that are purchased more frequently. The index was used to estimate ARDL and ARMA models to forecast 12-month inflation expectations, the CCI and the CPI. The index showed a good predictive capability to forecast the CPI for the low-income population and 12-month inflation expectations. When comparing the CPI and the IPPC, the relative difference between both tends to be bigger for higher levels of inflation. In 
addition, weekly purchased goods and services, such as gasoline, casados and taxi transport have a greater influence on the average of the monthly inter-annual percentage change of the IPPC, while the value of goods purchased biannually or annually is virtually zero.

KEY WORDS: PERCEPTION; PURCHASE FREQUENCY; MONETARY POLICY; CENTRAL BANK.

\section{INTRODUCCIÓN}

En la crisis de la década de los ochentas y durante los siguientes 20 años, la economía costarricense presentó una tasa de inflación de dos dígitos. A partir de 2009, el nivel de inflación ha sido menor; inclusive hasta alcanzar los rangos meta propuestos por el Banco Central de Costa Rica (ver gráfico 1). En particular, Torres (2012) determina la existencia de un cambio estructural para la serie de la variación interanual del IPC a partir de mayo de 2009, afirmando que "los resultados apuntan a que el comportamiento consistentemente bajo de la tasa de inflación interanual desde mayo de 2009 ha sido suficiente como para que las pruebas estadísticas detecten un cambio estructural significativo en su comportamiento en esa fecha" (Torres, 2012, p. 12).

\section{GRÁFICO 1 \\ COSTA RICA: VARIACIÓN INTERANUAL DEL IPC \\ JULIO 2007 - DICIEMBRE 2012}

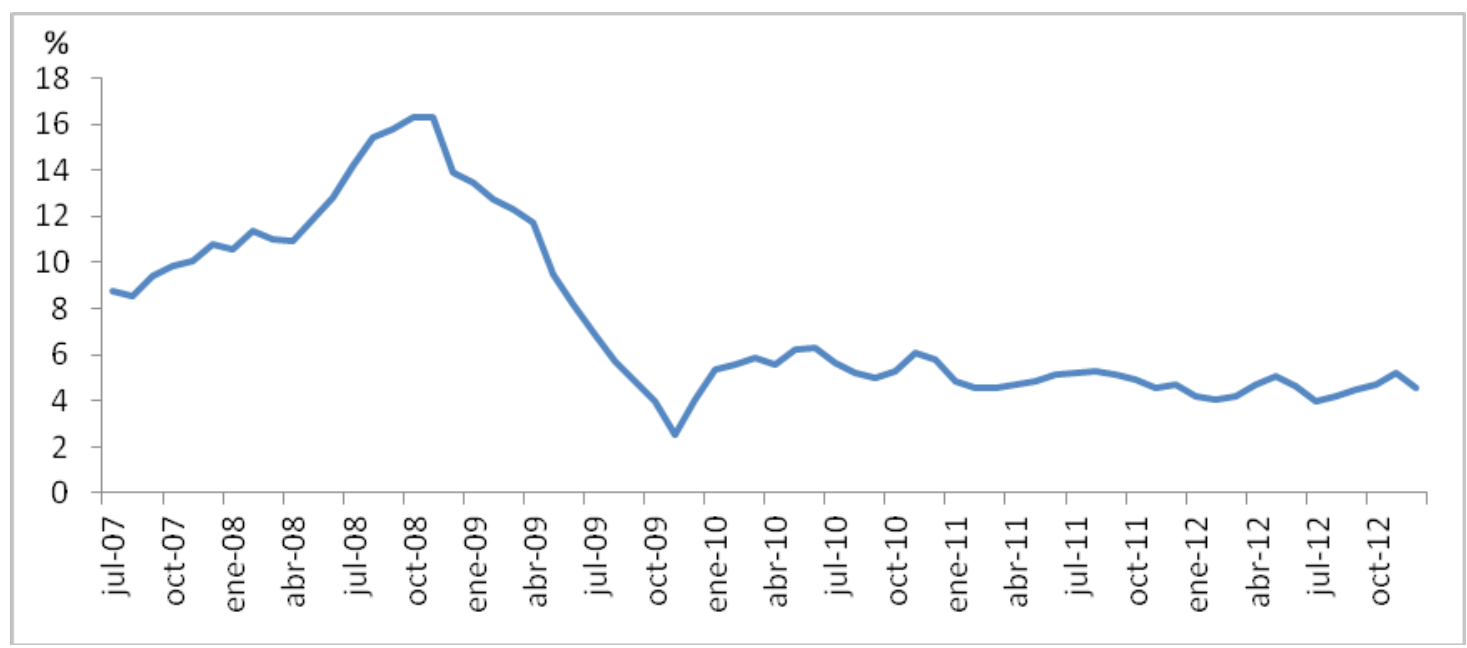

Fuente: elaboración propia con datos del INEC.

A pesar del cambio en el nivel de inflación para el 2009, uno de los descontentos más grandes de la población costarricense es el aumento en los precios de los bienes y servicios que consumen. Según la nota periodística publicada por La Nación el 12 de marzo de 2013 y a raíz de la última encuesta hecha por Unimer para el diario periodístico, el $21 \%$ de los costarricenses afirman que el desempleo es su principal desvelo, seguido por un $17 \%$ de la población que considera como su principal desasosiego el elevado costo de vida. 
Sin embargo, si las cifras oficiales muestran una tendencia a la baja en la inflación del país, ¿por qué la población sigue viéndola como uno de sus principales desvelos?

Es por este motivo que la presente investigación analiza si existe una diferencia estadísticamente significativa entre el IPC y el IPPC, de manera que se puedan justificar esas respuestas de los entrevistados por la encuesta de Unimer.

De acuerdo al artículo Inflación observada e inflación percibida en la zona del euro, publicado por el Banco Central Europeo ( 2007, pp. 64-75), la inflación percibida por los agentes puede ser distinta a la calculada por las autoridades según varias causas:

i) El consumidor suele centrarse en las compras frecuentes como alimentos y servicios básicos y se olvida de las compras esporádicas como viajes al exterior o automóviles.

ii) Se acuerda más de los incrementos de los precios que de las disminuciones, ya que las personas tienden a recordar las experiencias desagradables para no repetirlas en el futuro.

iii) Las percepciones sobre inflaciones altas son persistentes y suelen quedarse en la memoria a través del tiempo, por lo que generar cambios en las expectativas de la población implica un proceso gradual que podría tardar algunos años.

iv) No se tiene en cuenta los cambios en la calidad, los cuales implican aumentos en el valor, que deben verse como alteraciones en las características físicas de los productos que se adquieren y no como efecto simple de inflación.

v) Influencia de los medios de comunicación, que reflejan generalmente los cambios de los precios en bienes individuales y no necesariamente los de las cifras de inflación.

En este estudio se implementa por primera vez para Costa Rica un Índice de Inflación Percibida (IPPC), que se construye modificando el IPC con base en los fundamentos teóricos expuestos en los trabajos de Brachinger (2006, 2008). Se caracteriza, además, el comportamiento histórico y las propiedades estadísticas del IPPC, mientras que se utilizan los datos del índice para estimar modelos ARMA y ARDL para pronosticar las variables IPC, IPC-estrato alto, IPC-estrato medio, IPC-estrato bajo, IPC-bienes transables, IPC-bienes no transables, Índice de Confianza del Consumidor (ICC), y expectativas de inflación a 12 meses. La hipótesis que se plantea la investigación se basa en la premisa de que la inflación percibida es una variable relevante para la creación de expectativas de inflación; así como para determinar la confianza del consumidor. Por lo tanto, se logró demostrar que el IPPC puede servir para modelar expectativas, las cuales son cruciales para la formación de precios. Del mismo modo, el IPPC puede aportar información adicional relevante en la modelación de las variaciones de los precios del estrato de ingresos bajos, el cual se ve más expuesto y perjudicado ante los cambios en los precios.

\section{Marco teórico}

\section{Inflación percibida}

Siguiendo la línea de Kapounek y Lacina (2011), se debe tener claro que el término "percepción" se enmarca en la disciplina de la psicología. Según citan los autores, para la Oficina Federal de Estadística de Alemania, la percepción es el proceso de adquirir, interpretar, seleccionar y organizar la información sensorial; de modo que, para nuestro interés, el Índice de Inflación Percibida muestra la forma en que la población interpreta y organiza la información de los precios recibidos por parte del mercado. 
Los principales desarrollos teóricos sobre inflación percibida han sido formulados por Brachinger $(2006,2008)$. De acuerdo con el autor, existe una gran dificultad para medir la verdadera inflación, de manera que el desarrollo de cualquier índice de precios requiere de un marco interpretativo. Igualmente, argumenta que existen dos diferentes marcos interpretativos de inflación:

- La perspectiva del consumidor: bajo esta perspectiva lo que realmente importa es el consumo de ciertos bienes durante un periodo específico y son de particular interés los cambios de los precios de los bienes consumidos. Es decir, un bien tiene mayor relevancia para una persona mientras más grande es su proporción dentro del costo total destinado al consumo. El énfasis de esta perspectiva se centra en los costos incurridos por un consumidor promedio en un periodo reportado, a través de la adquisición de productos en una canasta de mercado.

- La perspectiva del comprador: desde este punto de vista, el agente se enfoca en las compras de determinados bienes en un periodo específico. Las personas muestran un especial interés en los cambios de precios de los bienes adquiridos en un periodo reciente. Desde esta perspectiva, resulta más significativo medir la frecuencia con que las compras son realizadas, que analizar el costo de una canasta de bienes en particular; puesto que un bien es más importante cuanto mayor es su frecuencia de compra.

Siguiendo a Brachinger $(2006,2008)$, se asume que cualquier intento de medir la inflación percibida tendrá que tomar la posición del comprador promedio y no del consumidor promedio, como frecuentemente se realiza. Por lo tanto, el punto de partida de las hipótesis en que se basa la teoría de la inflación percibida es la perspectiva del comprador. Dichas hipótesis se presentan a continuación.

- Primera hipótesis: plantea que en una fase preliminar de percepción el precio de cada bien es adquirido por el comprador, con un valor específico de referencia, aislando el resto de los productos y codificando esta comparación como una ganancia o pérdida, dependiendo de si el precio del bien es más bajo o más alto que el precio de su precio de referencia, respectivamente.

- Segunda hipótesis: plantea que cada cambio de precio es evaluado de acuerdo al valor que cada comprador le asigna a través de un función, que llamaremos V. En dicha función un incremento en el precio ("pérdida" para el comprador) se evalúa más que una disminución en el precio ("ganancia"para el comprador). Esto significa que el comprador percibe de manera más sensible un incremento del precio que una disminución en el mismo. Se asume, además, que esta evaluación asimétrica es realizada individualmente para cada cambio de precio que el consumidor enfrenta. Este tratamiento asimétrico de las ganancias y pérdidas es conocido como aversión a la pérdida.

- Tercera hipótesis: asume que los compradores forman sus juicios sobre la inflación, agregando mentalmente los cambios en el valor de los bienes que ellos recuerdan. Por lo tanto, la inflación se considera más alta si el comprador experimenta aumentos de precios de una forma más frecuente, haciendo así más fácil para él recordar los ejemplos notables de los aumentos de precios. En consecuencia, las reducciones de precios de productos pocas veces comprados, como la compra de tiquetes aéreos por ejemplo, o de los bienes que carecen de una acción explícita de compra, como los cargos automáticos, no tienen casi ningún efecto sobre la percepción de la inflación. En conclusión, la facilidad con que los aumentos de precios se recuerdan depende de la frecuencia con que los correspondientes bienes o servicios son comprados. 


\section{Trabajos empíricos sobre inflación percibida}

De los primeros estudios realizados respecto a la inflación percibida se encuentra Kahneman y Tversky (1973), donde se asegura que los agentes valoran la frecuencia de los eventos de acuerdo con la facilidad con que estos pueden ser recordados. Por lo tanto, la percepción de un cambio en un precio es más fuerte conforme más alta sea la frecuencia de compra de ese producto. Posteriormente en un estudio experimental, Kahneman y Tversky (1981) concluyeron que la percepción de los cambios individuales en los precios es $5 \%$ más fuerte para los bienes relativamente más baratos que para los bienes más caros.

Con respecto a los supuestos de Brachinger (2006, 2008), es importante señalar que varios autores se han dado a la tarea de probarlos, encontrando evidencia estadística a su favor. Por ejemplo, Georganas, Healy y Li (2014) determinaronn que los bienes que se compran con más frecuencia influyen en mayor medida en la inflación percibida por los consumidores; aun cuando estos bienes representan una parte relativamente pequeña del gasto total. Esto sugiere que va a tener mayor influencia la variación en el costo de productos como la gasolina y los alimentos; mientras que los bienes con baja frecuencia de compra, la vivienda por ejemplo, van a tener un peso menor.

Del mismo modo, los autores coinciden al proponer que hay correlación entre la percepción de la inflación y la diferencia existente entre las tasas de inflación de los bienes no duraderos y los duraderos. Dado que los primeros se compran con más frecuencia que los segundos, los consumidores pueden sesgar su percepción de la inflación de la economía hacia la tasa de inflación de los bienes comprados con mayor frecuencia. En otras palabras, los consumidores que experimentan altos aumentos de los precios de gasolina pueden concluir erróneamente que la inflación en toda la economía es alta.

De este modo se tiene que:

donde $\theta_{i t}=\frac{q_{i} p_{i t}}{\sum_{j} q_{j} p_{j t}}$,

$$
\Pi_{t}=\sum_{i} \theta_{i t} \pi_{i t}
$$

con $\theta_{i t}$ como el peso del gasto del bien $i$ en $t$.

Así, los consumidores pueden tener percepciones por cada bien $i$, denotada por $\pi_{i}^{p}$, que podría diferir o no de la tasa de variación de precio dada para el bien $i, \pi_{i}$. Por tanto, para el total de la economía, existe una diferencia entre la tasa de inflación percibida y la dada. De este modo, se plantea la tasa de inflación percibida de manera agregada como:

$$
\Pi^{p}=\sum_{i} w_{i} \pi_{i}^{p}
$$

Donde $w_{i}$ es el peso que el consumidor pone en $\pi_{i}^{p}$. De esta manera, independientemente de la exactitud de cada $\pi_{i}^{p}$, si un consumidor entiende que las tasas de inflación fueron calculadas por la construcción de una canasta de $q_{i}$ unidades de cada bien $i$, se debe tener que $w_{i}=\theta_{i}$ para cada bien $i$.

Por ende, cuando los pesos reales de los consumidores $w_{i}$ se desvían de $\theta_{i}$, con más peso en los bienes que se compran con mayor frecuencia y menor peso en aquellos bienes que se compran con menor frecuencia, se genera un sesgo en la inflación percibida. Para lograr separar la frecuencia de compras de la cantidad comprada, los autores definen: 


$$
q_{i}=d_{i} \mu_{i}
$$

donde $d_{i}$ es el número de veces que el bien $i$ fue comprado en el periodo dado y $\mu_{i}$ es el promedio de la cantidad por compra.

El peso de la frecuencia de consumo es dada por:

$$
\varphi_{i}=\frac{d_{i}}{\sum_{j} d_{j}}
$$

A partir de estos pesos definen formalmente el sesgo generado en la percepción de la inflación por parte de la frecuencia de consumo.

La percepción de la inflación del consumidor que muestra el sesgo de la frecuencia, si la hay, es cierta para $\alpha>0$, de manera tal que para cada bien $i$ :

$$
w_{i}=\alpha \varphi_{i}+(1-\alpha) \theta_{i}
$$

donde:

$$
\begin{aligned}
& \text { - } \varphi_{i}=\frac{d_{i}}{\sum_{j} d_{j}} \text {, es la frecuencia relativa para cada bien comprado. } \\
& \text { - } \theta_{i}=\frac{P_{i}}{P} \text {, es la razón del total de gastos realizados en el bien . }
\end{aligned}
$$

El grado en que cada consumidor utiliza el peso de las frecuencias versus el peso del gasto es capturado por el parámetro $\alpha$. Así, un consumidor imparcial tiene $\alpha .=0$. Por ello, dado $\alpha$, la percepción de la inflación general es calculada como:

$$
\begin{gathered}
\Pi^{P}=\sum_{i} w_{i} \pi_{i}^{P} \\
=\sum_{i}\left[\alpha \varphi_{i}+(1-\alpha) \theta_{i}\right] \pi_{i}^{P}
\end{gathered}
$$

Al tener $\Pi_{\text {gasto }}^{\mathrm{P}}=\sum_{\mathrm{i}} \theta_{\mathrm{i}} \pi_{\mathrm{i}}^{\mathrm{P}}$ como la tasa de inflación del correcto gasto ponderado $y$

$$
\begin{gathered}
\Pi_{\text {frec }}^{\mathrm{P}}=\sum_{\mathrm{i}} \varphi_{\mathrm{i}} \pi_{\mathrm{i}}^{\mathrm{P}} \text { como la tasa de inflación basada en la frecuencia de consumo, se obtiene: } \\
\Pi^{P}=\alpha \Pi_{\text {frec }}^{P}+(1-\alpha) \Pi_{\text {gasto }}^{P}
\end{gathered}
$$

Romina (2007) argumenta que las compras frecuentes reflejan fielmente las percepciones de los consumidores respecto a la inflación. En efecto, ese estudio evidencia que a medida que los cambios en el índice de compras frecuentes aumentan más que el índice de precios promedio de la economía, la inflación percibida por parte de los consumidores es mayor. Las pruebas econométricas realizadas 
por Romina utilizando el Índice de Inflación Percibida para Malta, el Índice de Precios al Consumidor armonizado de la Unión Europea y el balance estadístico de inflación percibida -PIBS por sus siglas en inglés- muestran además que el Índice de Inflación Percibida para Malta y el PIBS se mueven paralelamente, mientras que la relación entre el índice de precios armonizado y el Índice de Inflación Percibida para Malta no evidencia un nivel fuerte de significancia. Esto sugiere que la percepción de la inflación según los consumidores de Malta está en línea con la evolución de los precios de los bienes $y$ servicios adquiridos con mayor frecuencia y no con la medida por el índice de precios armonizado.

Haschka (2004), en su trabajo sobre la economía austriaca, mostró que el índice de precios conformado por bienes comprados diariamente se incrementó en promedio un 3,3\% del 2001 al 2004, mientras que otro conformado por compras semanales se incrementó en 2,5\%. Por el contrario, el IPC solo presentó un incremento del $2 \%$. Además, concluye que la proporción de bienes que experimentaron incrementos de precio en ese mismo periodo fue más alta para los bienes comprados en forma diaria o semanal que para el resto de los bienes que conforman la canasta del IPC.

Por otra parte, Fritsche, Vogel y Menz (2009) realizaron un cuidadoso análisis sobre los supuestos de Brachinger (2006, 2008). Los autores investigaron la posibilidad de que los puntos de vista sobre el comportamiento económico, notablemente la teoría de las expectativas, puedan ser aplicadas para proveer explicaciones sobre la formación individual de la percepción de la inflación. En este caso, los autores utilizaron un modelo dinámico de panel para 12 países europeos para el periodo entre enero de 1996 y noviembre de 2008, en el cual encontraron evidencia empírica de dos de las hipótesis utilizadas por Brachinger en su Índice de Inflación Percibida.

En primer lugar, encontraron evidencia de "aversión a las pérdidas" en los hogares para toda la muestra seleccionada de países, en el periodo anterior a la introducción del euro. En segundo lugar, determinaron que la inflación de los bienes que se adquieren con mayor frecuencia tiene un efecto significativo en la inflación percibida para toda la muestra seleccionada; mientras que las tasas de variación de precio de otras categorías de bienes no tienen un efecto importante. En la época posterior a la introducción del euro, también se encontró que la categoría de bienes comprados de manera más frecuente tenían un mayor efecto en la percepción de la inflación. Otro hallazgo de los autores es que la percepción de los cambios individuales en los precios es más fuerte conforme un bien es comprado con más frecuencia, para lo que ya Kahneman y Tversky (1981) identificaron evidencia en su estudio experimental.

En conclusión, existe evidencia empírica que apoya la validez de los supuestos planteados por Brachinger (2006, 2008). El enfoque de la percepción de la inflación corresponde exclusivamente a los cambios de precios, por lo tanto, se tiene en cuenta que los cambios en la percepción podrían ser inducidos por modificaciones en las frecuencias de compra.

\section{Formulación de expectativas en Costa Rica}

Para Costa Rica, según Alfaro y Monge (2013), en el proceso de formación de expectativas de inflación para el periodo entre enero de 2006 y diciembre de 2013, es posible rechazar la hipótesis de expectativas racionales para todos los agentes consultados, sin embargo también es necesario dejar claro que la persistencia en los errores tampoco implica que el proceso tenga una relación tan solo con la inflación pasada, como sugeriría el proceso de formación de expectativas adaptativas, esto se debe a que los agentes de cierto modo incorporan alguna información macroeconómica disponible para la formulación de las expectativas. 


\section{METODOLOGÍA}

La propuesta metodológica de la investigación para la construcción del Índice de Precios Percibidos del Consumidor toma como base la metodología del IPC base julio del 2006 y realiza modificaciones, utilizando la teoría sobre inflación percibida propuesta por Brachinger (2008). Una vez construido el IPPC, se analiza su comportamiento histórico y sus propiedades estadísticas, en comparación con la inflación calculada por el INEC. Con base en el cálculo de la inflación percibida por el comprador, se utilizan modelos de series de tiempo ARMA y ARDL para pronosticar el ICC, las Expectativas de Inflación a 12 meses, el IPC y sus distintas variantes. Por último, se evalúa los pronósticos con pruebas de insesgamiento, capacidad de pronóstico y capacidad de predecir dirección de movimiento en variable de interés.

\section{Cálculo del IPPC}

Con respecto a los criterios establecidos para la construcción del IPC base julio del 2006, se destaca que el IPPC conserva la mayoría de los principios; entre ellos: fuente de información, cobertura geográfica, periodo base, población de referencia, clasificación de los bienes y servicios de la canasta y niveles de agregación de los bienes y servicios. Con esto, se asegura la representatividad y la cobertura geográfica del mismo, dos criterios de suma importancia en la construcción de cualquier índice.

\section{Ponderación de los bienes y servicios del IPPC}

De acuerdo con Brachinger (2008), existen dos perspectivas para comprender la inflación: desde la visión del comprador promedio y desde la del consumidor promedio. Se asume que al medir la inflación percibida se toma la posición del comprador promedio y no del consumidor promedio. Sin embargo, en este artículo la perspectiva del consumidor también se toma en cuenta, pues se considera que al tomar como punto de partida tanto los bienes y servicios como su peso, la canasta en la construcción del IPPC, se complementa ambas perspectivas para hacer más robusto el indicador.

En la canasta del IPC base julio 2006, se incluyen bienes o servicios cuyo peso en el gasto de consumo de cada familia sea al menos $0,05 \%$, o que sean consumidos por al menos el $5 \%$ de los hogares. Esto resultó en 225 bienes y 67 servicios, que representan el 88,5\% del gasto reportado por el total de los hogares del área de cobertura del IPC.

Sin embargo, emplear únicamente estos dos criterios presenta una limitación: ambos factores están más alineados con la perspectiva del consumidor, dejando de lado la importancia de la frecuencia de las compras de los bienes y servicios. Por tanto, con el fin de tomar como marco interpretativo de la inflación la perspectiva del comprador, se debe incluir un criterio adicional para calcular el IPPC. Este consiste en darle mayor peso a los bienes y servicios adquiridos con mayor frecuencia por las familias. Esto se realiza, porque en la canasta de consumo del IPC base julio 2006 se incluyen bienes y servicios que no son regularmente adquiridos por la mayoría de los hogares. Por ejemplo, algunos bienes o servicios incluidos podrían tener una frecuencia de compra menor a los 2 o 3 años. Esto podría generar que las familias no perciban de igual manera cambios en los precios de estos artículos y que por ende no sean relevantes para su percepción de la inflación.

La frecuencia de compra de los bienes y servicios se obtuvo de la Metodología de la Encuesta Nacional de Ingresos y Gastos de los Hogares (ENIG) 2004 (INEC, 2006), a partir de su clasificación de los gastos según período de referencia. La clasificación que se realiza de los gastos en la ENIG 2004 según período de referencia es: 
- Gastos diarios

- Gastos mensuales

- Gastos trimestrales

- Gastos semestrales

- Gastos anuales

Es indispensable aclarar que cuando se habla de gastos diarios no implica que se compren los bienes día a día y se consuman, sino que son bienes o servicios que se pueden adquirir cualquier día del mes, sin importar su fecha de consumo. Ante esto, para efectos de la presente investigación, el término gastos diarios se toma como gastos que se realizan de forma semanal.

Respecto al IPC, resulta indispensable entender el método de ponderación de los bienes y servicios que componen dicho índice, de modo que se pueda comprender más fácilmente la nueva ponderación propuesta. Así la fórmula de cálculo de la ponderación de cada bien en el IPC es:

$$
w_{i}=\frac{\sum_{h=1}^{n} g_{i h}^{0}}{\sum_{i=1}^{292}\left(\sum_{h=1}^{n} g_{i h}^{0}\right)}
$$

donde:

$w_{i}=$ ponderación del artículo $i$

$g_{i}^{0}=$ gasto en el artículo $i$ en el periodo de referencia

$i=$ artículos

$h=$ hogar

$n$ = cantidad de hogares

Tomando esto en cuenta, se calcula el IPPC de dos maneras distintas:

\section{IPPC 1}

La reponderación de los bienes y servicios se calcula tomando en cuenta la cantidad de veces que el comprador se expone al precio por año, con el objetivo de darle más importancia a los precios de los bienes a los que se exponen los agentes frecuentemente. Se considera que estos precios son los que, en teoría, los compradores lograrían recordar con mayor facilidad a la hora de formar su percepción. Para identificar la frecuencia relativa con la que el comprador se enfrenta a cada precio, la ponderación se calcula de la siguiente manera:

$$
w_{i}^{I P P C 1}=w_{i}^{I P C} \cdot\left(\frac{1}{x}\right)
$$


donde:

$$
\begin{array}{ll}
w_{i}^{I P P C 1} & =\text { ponderación final del artículo i para el IPPC1. } \\
w_{i}^{I P C} & =\text { ponderacion inicial del IPC. } \\
x & =\text { número de semanas del periodo de referencia } .
\end{array}
$$

De este modo se tiene que:

- Frecuencia de compra semanal:

$$
w_{i}^{I P P C 1}=w_{i}^{I P C} \cdot 1=w_{i}^{I P C}
$$

Por ende la ponderación de estos artículos permanece intacta.

- Frecuencia de compra mensual:

$$
w_{i}^{I P P C 1}=w_{i}^{I P C} / 4
$$

Las ponderaciones iniciales de estos artículos se dividen entre 4.

- Frecuencia de compra trimestral:

$$
w_{i}^{I P P C 1}=w_{i}^{I P C} / 12 \text {. }
$$

Las ponderaciones iniciales de estos artículos se dividen entre 12.

- Frecuencia de compra semestral:

$$
w_{i}^{I P P C 1}=w_{i}^{I P C} / 24
$$

Las ponderaciones iniciales de estos artículos se dividen entre 24.

- Frecuencia de compra anual:

$$
w_{i}^{I P P C 1}=w_{i}^{I P C} / 52
$$

Las ponderaciones iniciales de estos artículos se dividen entre 52.

Por último, se reescalan las nuevas ponderaciones para que vuelvan a sumar 1 .

\section{$I P P C 2$}

La reponderación de los bienes y servicios se calcula tomando en cuenta la cantidad de veces que el comprador se expone al precio por año, con el objetivo de darle más importancia a los precios de los bienes a los que se exponen los agentes frecuentemente. Se considera que estos son los que, en teoría, los compradores lograrían recordar con mayor facilidad a la hora de formar su percepción. Para identificar la frecuencia relativa con la que el comprador se enfrenta a cada precio, la ponderación se calcula de la siguiente manera:

$$
w_{i}^{I P P C 2}=w_{i}^{I P C} \cdot\left(\frac{y_{j}}{\sum_{j=1}^{5} y_{j}}\right)
$$


donde:

$w_{i}^{I P P C 2}=$ ponderación final del artículo $i$ para el IPPC1.

$w_{i}^{I P C}=$ ponderacion inicial del IPC.

$y_{j} \quad$ = número de semanas del periodo de referencia .

De este modo se tiene que:

- Frecuencia de compra semanal:

$$
w_{i}^{I P P C 2}=w_{i}^{I P C} \cdot\left(\frac{y_{1}}{\sum_{j=1}^{5} y_{j}}\right)=w_{i}^{I P C} \cdot\left(\frac{52}{(52+12+4+2+1)}\right)=w_{i}^{I P C} \cdot\left(\frac{52}{71}\right)=w_{i}^{I P C} \cdot(0,732)
$$

Las ponderaciones iniciales de estos artículos se multiplican por 0,732.

- Frecuencia de compra mensual:

$$
w_{i}^{I P P C 2}=w_{i}^{I P C} \cdot\left(\frac{12}{71}\right)=w_{i}^{I P C} \cdot(0,169)
$$

Las ponderaciones iniciales de estos artículos se multiplican por 0,169.

- Frecuencia de compra trimestral:

$$
w_{i}^{I P P C 2}=w_{i}^{I P C} \cdot\left(\frac{4}{71}\right)=w_{i}^{I P C} \cdot(0,056)
$$

Las ponderaciones iniciales de estos artículos se multiplican por 0,056.

- Frecuencia de compra semestral:

$$
w_{i}^{I P P C 2}=w_{i}^{I P C} \cdot\left(\frac{2}{71}\right)=w_{i}^{I P C} \cdot(0,028)
$$

Las ponderaciones iniciales de estos artículos se multiplican por 0,028.

- Frecuencia de compra anual:

$$
w_{i}^{I P P C 2}=w_{i}^{I P C} \cdot\left(\frac{1}{71}\right)=w_{i}^{I P C} \cdot(0,014)
$$

Las ponderaciones iniciales de estos artículos se multiplican por 0,014.

Finalmente, se reescalan las nuevas ponderaciones para que vuelvan a sumar 1.

Una vez completada la reponderación de los bienes y servicios de la nueva canasta, se calcula el IPPC con las series de precios históricos utilizadas por el INEC para el cálculo del IPC. El período base del IPPC es también julio del 2006, y su cálculo se realiza hasta diciembre del 2012. Asimismo, se calculan las variaciones mensuales e interanuales. Además, ya que el IPPC está basado en un índice de tipo Laspeyres, puede ser directamente comparado con el IPC por ser un caso especial de la misma familia de los índices de precios. Para dicha comparación, se utilizan la media, mediana, desviación estándar, coeficiente de variación, máximo y mínimo; al igual que el análisis gráfico. Por último, se calcula la participación de los grupos y artículos, así como la participación de la periodicidad de compra en la media de la variación interanual mensual de ambos índices. 


\section{Cálculo de los pronósticos}

Se pronostican las variables IPC, IPC-estrato alto, IPC-estrato medio, IPC-estrato bajo ${ }^{4}$, IPC-bienes transables, IPC-bienes no transables, ICC y expectativas de inflación a 12 meses mediante dos métodos. En primer lugar, se utiliza modelos ARMA (p,q) (Autorregresivos de Medias Móviles), que no consideran información de la medida de inflación percibida. En segundo lugar, utiliza modelos ARDL (Autorregresivos de Rezagos Distribuidos), en los cuales el modelo va a ser explicado por sus propios valores en periodos anteriores y, además, por los valores pasados de la medida de inflación percibida.

Para los modelos ARMA, la especificación se escogió mediante la metodología de Box- Jenkins, mientras que en los modelos ARDL se utilizó el principio de búsqueda de especificación "general a específico", propuesto por Hendry (1979). Las especificaciones estimadas se evalúan mediante indicadores de ajuste, pruebas de significancia de los coeficientes, normalidad de los errores (Jarque-Bera) y estacionariedad al modelo.

Los datos utilizados son:

- Expectativas de inflación a 12 meses: variaciones interanuales, frecuencia mensual, julio-2007 -diciembre- 2012.

- Variaciones ICC: Trimestrales, frecuencia trimestral, 2006-IV - 2012-IV.

- Para las restantes series (IPC y sus variantes) se utilizarán interanuales, empezando en agosto del 2006 y finalizando en diciembre del 2012.

Se calcula pronósticos un paso adelante. Se seleccionó una muestra de estimación creciente (inicio fijo) a la hora de realizar cada pronóstico, según la cual en cada período se reestiman los parámetros del modelo y se proyecta las variables de interés un período hacia adelante. Se generan pronósticos para el periodo julio 2011-diciembre 2012, para un total de 18 observaciones para cada serie (a excepción de la serie del ICC, para el que se generan 6 observaciones).

La muestra de estimación en el caso de las variaciones interanuales de las expectativas de inflación a 12 meses es julio-2007 y junio-2011 (48 periodos); mientras que la muestra de pronóstico y evaluación abarca los últimos 18 periodos de la serie (julio 2011 a diciembre 2012). Para las series de variaciones interanuales del IPC y sus diferentes variantes, se utilizará el mismo criterio, por lo que la muestra de estimación es agosto-2006 a junio-2011 (59 periodos) y la muestra de evaluación es julio-2011 a diciembre-2012 (18 periodos). Para el caso de las variaciones trimestrales del ICC, se realiza la misma selección; sin embargo, por la diferente temporalidad de la serie:

- $\quad$ muestra de estimación es 2006-IV a 2011-II (19 periodos).

- $\quad$ muestra de evaluación es 2011-III a 2012-IV (6 periodos).

4 La definición de los estratos se realiza por parte del INEC, ver página 5 del documento: http://www.inec.go.cr/sites/default/files/documetos-biblioteca-virtual/ meeconomipcbasejulio2006-03.pdf 


\section{Evaluación de los pronósticos}

Para determinar cuál modelo presenta una mejor capacidad de pronóstico, se realizan pruebas a cada serie de pronósticos (ARMA-ARDL). Los criterios de evaluación son los siguientes:

a) Insesgamiento: En el caso de una serie de pronósticos, se dice que $\hat{y}_{\mathrm{t}}$ es un estimador insesgado de $\mathrm{y}_{\mathrm{t}}$ si el valor esperado de $\hat{\mathrm{y}}_{\mathrm{t}}$ es igual al valor observado de $\mathrm{y}_{\mathrm{t}}$, es decir:

$E\left(\hat{\mathrm{y}}_{\mathrm{t}}\right)=E\left(\mathrm{y}_{\mathrm{t}}\right) \circ E\left(\hat{\mathrm{y}}_{\mathrm{t}}\right)-E\left(\mathrm{y}_{\mathrm{t}}\right)=0$.

Para comprobar esto, se estima una ecuación donde:

$$
y_{t}=\alpha+\beta \hat{y}_{t}+u_{t}
$$

donde:

$$
\begin{aligned}
& \mathrm{y}_{\mathrm{t}}=\text { Valor observado. } \\
& \hat{\mathrm{y}}_{\mathrm{t}}=\text { Valor pronosticado. } \\
& \mathrm{u}_{\mathrm{t}}=\text { Termino de error. }
\end{aligned}
$$

Para que la serie de pronósticos sea insesgada (para que $\quad E\left(\hat{\mathrm{y}}_{\mathrm{t}}\right)=E\left(\mathrm{y}_{\mathrm{t}}\right)$ ), los parámetros $\alpha y \beta$, debe ser 0 y 1 , respectivamente, por lo que el insesgamiento se comprueba si no se rechaza la hipótesis conjunta $\alpha=0$ y $\beta=1$ (Holden y Peel, 1989).

b) Indicadores de ajuste: Se calculó la raíz error cuadrático medio (RECM), la cual es la raíz del promedio de los errores de pronóstico cuadrados. Suponiendo que la muestra de pronósticos es $\mathrm{j}=\mathrm{T}+1, \mathrm{~T}+2, \ldots, \mathrm{T}+\mathrm{h}, \mathrm{y}$ denotando que el valor actual $\mathrm{y}$ el valor pronosticado en el periodo es $y_{t} y \hat{y}_{t}$, respectivamente, el RECM se calcula de la siguiente manera:

$$
R E C M=\sqrt{\sum_{j=T+1}^{T+h}\left(\hat{y}_{t}-y_{t}\right)^{2} / h}
$$

c) Capacidad de pronóstico: la prueba de capacidad de pronóstico utilizada fue la propuesta por Diebold y Mariano (1995), la cual compara dos pronósticos para una serie y permite establecer si uno de ellos incorpora toda la información relevante del pronóstico alternativo, partiendo de una hipótesis nula de igual poder predictivo. (Diebold \& Mariano, 1995). Se utiliza la variante basada en el ECM.

d) Capacidad de predecir dirección de movimiento en variable de interés: la predicción de la dirección de la variable de interés (signo de la tasa de variación) se mide calculando cuál porcentaje de los signos se predijeron correctamente. 


\section{RESULTADOS}

Análisis de los índices de precios percibidos al comprador

La evolución de las dos variantes del IPPC es prácticamente la misma, pero sí existen diferencias con respecto al IPC. Como se aprecia en el cuadro 1, en promedio la inflación percibida se ubica por encima de la inflación calculada por el INEC. Asimismo, parece darse un cambio estructural en el IPPC, compatible con el quiebre estructural para la serie de inflación agregada, propuesto por Torres (2012), (ver gráfico 2).

CUADRO 1

ESTADÍSTICAS DESCRIPTIVAS DE LA VARIACIÓN INTERANUAL JULIO 2007 - DICIEMBRE 2012

\begin{tabular}{lrrr}
\hline Estadísticos & IPC & IPPC 1 & IPPC 2 \\
\hline Media & 7,49 & 8,72 & 8,73 \\
Mediana & 5,58 & 6,30 & 6,32 \\
Desv. Estándar & 3,72 & 5,33 & 5,37 \\
Coef. Variación & 49,71 & 61,12 & 61,45 \\
Máximo & 16,30 & 21,33 & 21,43 \\
Mínimo & 2,57 & $-0,25$ & $-0,32$ \\
No. Observaciones & 66 & 66 & 66 \\
\hline
\end{tabular}

Fuente: Elaboración propia con datos propios y del INEC.

GRÁFICO 2

COSTA RICA: COMPORTAMIENTO TASA DE

VARIACIÓN INTERANUAL IPPC1, IPPC2

JULIO 2007 - DICIEMBRE 2012

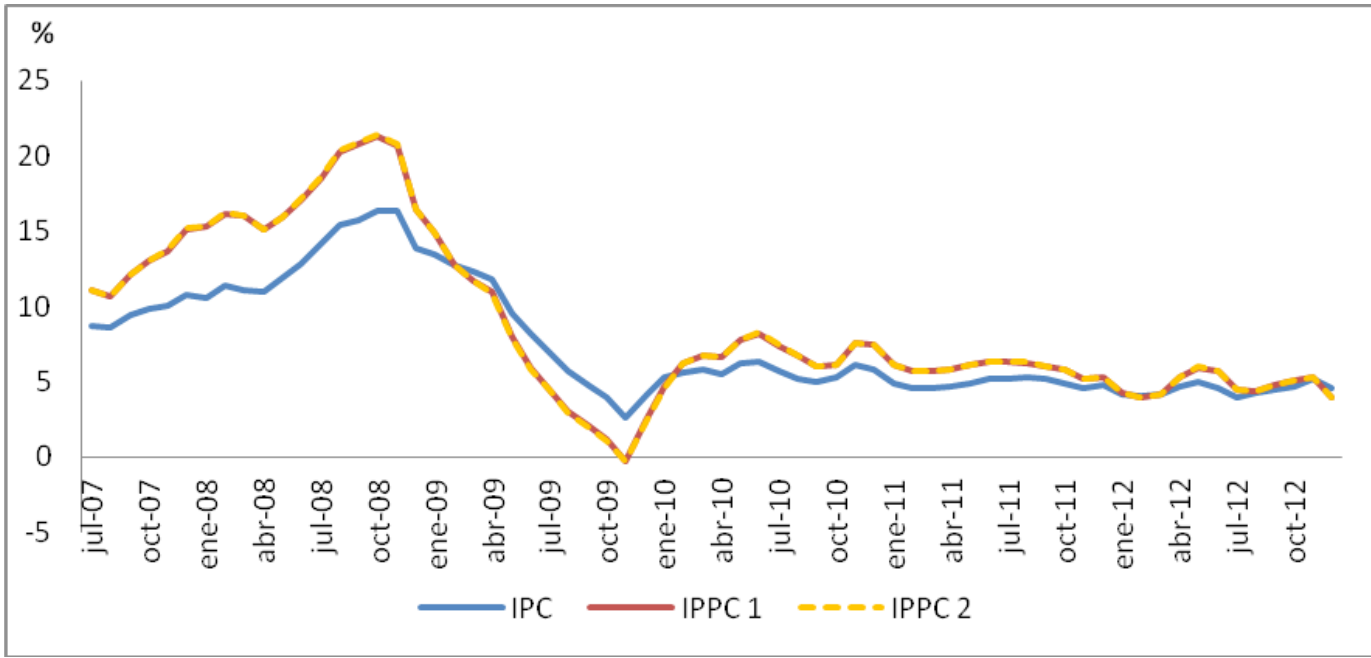

Fuente: Elaboración propia. 
Al analizar los componentes del IPPC, la participación de los grupos "Alimentos y bebidas no alcohólicas", "Transporte" y "Comidas y bebidas fuera del hogar" es mayor en la media de la variación interanual mensual del IPPC en comparación con el IPC. Esto refleja la importancia de los productos consumidos con mayor frecuencia. Resulta razonable que artículos como la gasolina, el casado y el transporte en taxi tengan una importancia relativa mayor en comparación con la que tienen en el IPC. Artículos como el casado, el servicio de electricidad y el servicio doméstico son los que más peso tienen.

GRÁFICO 3

PARTICIPACIÓN DE LOS GRUPOS EN LA MEDIA DE LA VARIACIÓN INTERANUAL

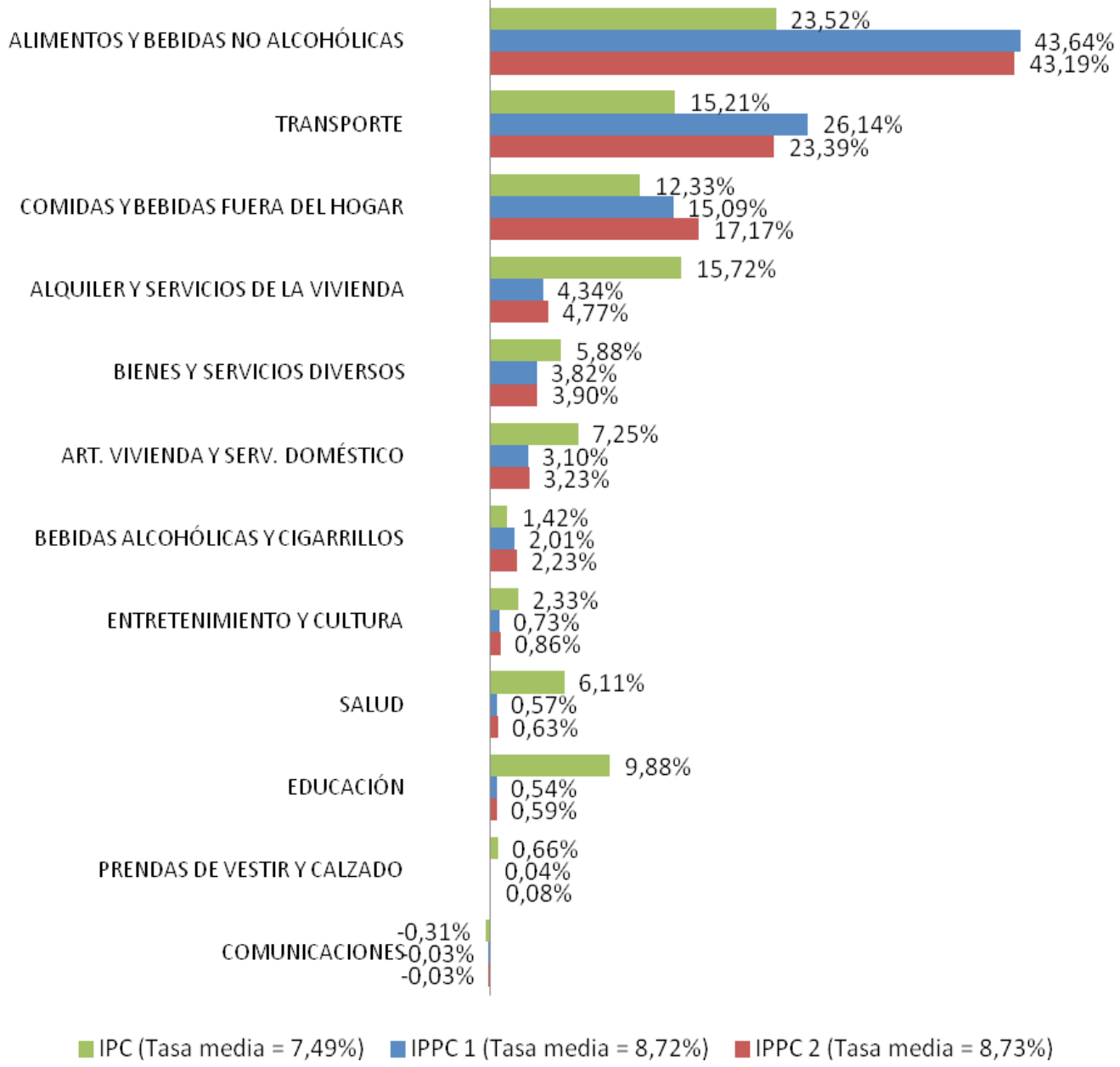

Fuente: Elaboración propia con datos de INEC.

Con respecto a la periodicidad de compra, los artículos que más influyen en la media de la variación interanual del IPPC son los comprados semanalmente, con un aporte superior al 90\%. La participación disminuye al considerar sucesivamente los bienes de consumo mensual, trimestral y semestral, para terminar con una participación prácticamente nula de los de consumo anual. En comparación, en el IPC, los bienes semanales solo aportan el aproximadamente el $52 \%$ a la media de la variación. 
CUADRO 2

PARTICIPACIÓN DE LA PERIODICIDAD DE COMPRA EN LA MEDIA DE LA VARIACIÓN INTERANUAL MENSUAL AGOSTO 2006 - DICIEMBRE 2012

\begin{tabular}{lccc}
\hline & IPC & IPPC 1 & IPPC 2 \\
\hline Anual & $2,06 \%$ & $0,08 \%$ & $0,08 \%$ \\
Semestral & $15,85 \%$ & $0,80 \%$ & $0,84 \%$ \\
Trimestral & $7,58 \%$ & $0,65 \%$ & $0,75 \%$ \\
Mensual & $22,98 \%$ & $6,86 \%$ & $7,37 \%$ \\
Semanal & $51,52 \%$ & $91,61 \%$ & $90,95 \%$ \\
TOTAL & $100,00 \%$ & $100,00 \%$ & $100,00 \%$ \\
\hline
\end{tabular}

Fuente: Elaboración de los autores con datos propios y del INEC.

Capacidad de pronóstico de variables de interés

Después de realizar el proceso de selección para los modelos ARMA y ARDL ${ }^{5}$, las especificaciones seleccionadas son las siguientes:

CUADRO 3

MODELOS SELECCIONADOS PARA CADA SERIE

\begin{tabular}{lcc}
\hline Serie & $\begin{array}{c}\text { Especificación } \\
\text { ARMA }\end{array}$ & Especificación ARDL \\
\hline IPC & ARMA $(2,4)$ & $I P C_{t-1} I P P C_{t-1} I P P C_{t-5}$ \\
IPC-estrato alto & ARMA $(2,1)$ & $I P C_{-} E A_{t-12} I P P C_{t-1} I P P C_{t-5}$ \\
IPC-estrato medio & ARMA $(1,2)$ & $I P P C_{t-1} I P P C_{t-5}$ \\
IPC-estrato bajo & ARMA $(1,1)$ & $I P C_{-} E B_{t-1} I P P C_{t-5}$ \\
IPC-bienes transables & ARMA (3,4) & $I P C_{-} T_{t-1} I P C_{-} T_{t-6} I P P C_{t-5}$ \\
IPC-bienes no transables & ARMA $(2,2)$ & $I P C_{-} N T_{t-12} I P P C_{t-1} I P P C_{t-3} I P P C_{t-5} I P P C_{t-6}$ \\
ICC & ARMA (4,3) & $I C C_{t-6} I P P C_{t-10}$ \\
Expectativas de inflación a 12 meses & ARMA (3,1) & $\operatorname{Exp}_{t-1}^{12} \operatorname{Exp}_{t-9}^{12} I P P C_{t-1} I P P C_{t-9} I P P C_{t-11}$ \\
\hline
\end{tabular}

Fuente: Elaboración propia.

$5 \quad$ No existe una diferencia estadísticamente significativa entre las dos ponderaciones del IPPC y por eso es irrelevante entre cual se utilice, de este modo se utiliza el IPPC1 como base para hacer los pronósticos. 
Los modelos seleccionados fueron los que mejor se ajustaron a los datos, cumpliendo con todos los análisis y pruebas realizadas. No obstante, esto no los libra de tener limitaciones, las cuales -en el caso de cada especificación de los modelos ARMA- son las siguientes:

CUADRO 4

LIMITACIONES DE LAS ESPECIFICACIONES SELECCIONADAS

PARA LOS MODELOS ARMA

\begin{tabular}{ll}
\hline \multicolumn{1}{c}{ Serie } & \multicolumn{1}{c}{ Limitaciones } \\
\hline IPC & Bondad de ajuste muy baja \\
IPC-estrato alto & Coeficiente de AR(2) no es significativo \\
IPC-estrato medio & - \\
IPC-estrato bajo & Coeficiente de AR(1) no es significativo \\
IPC-bienes transables & Coeficiente de AR(3) no es significativo \\
IPC-bienes no transables & \\
ICC & Coeficiente de MA(3) no es significativo \\
Expectativas de inflación a 12 meses & Coeficiente de MA(1) no es significativo \\
\hline
\end{tabular}

Fuente: Elaboración propia.

A pesar de que la mayoría de los modelos seleccionados tienen como limitación que alguno de los coeficientes es no significativo, se utilizan, ya que presentan un mejor ajuste que las restantes sugeridas por el algoritmo de selección. Además, cumplen con las propiedades evaluadas en las otras pruebas.

En el caso de las especificaciones seleccionadas para los modelos ARDL, las limitaciones son las siguientes:

\section{CUADRO 5}

LIMITACIONES DE LAS ESPECIFICACIONES SELECCIONADAS

PARA LOS MODELOS ARDL

\begin{tabular}{ll}
\hline Serie & Limitaciones \\
\hline IPC & Coeficiente de no es significativo \\
IPC-estrato alto & - \\
IPC-estrato medio & Ningún coeficiente del componente autorregresivo \\
& es significativo \\
IPC-estrato bajo & Se rechaza hipótesis de normalidad de los errores \\
IPC-bienes transables & Se rechaza hipótesis de normalidad de los errores \\
IPC-bienes no transables & - \\
ICC & Ningún rezago del es significativo \\
Expectativas de inflación a 12 meses & - \\
\hline
\end{tabular}

Fuente: Elaboración propia. 
Con respecto a las especificaciones seleccionadas para los ARDL, el caso de la serie IPC muestra la restricción que el coeficiente de $I P C_{t-1}$ no es significativo, aunque el mismo se incluye para que el modelo incluido en un componente autorregresivo. Para la serie IPC-estrato medio, la limitación que presenta es que ningún coeficiente del componente autorregresivo es significativo y su inclusión no mejora el desempeño del modelo, por lo que no se incluye. En el caso de las series IPC-estrato bajo e IPC-bienes transables, se rechaza la hipótesis de la distribución normal de los errores, lo que provoca que los intervalos de confianza no puedan utilizarse de manera usual y correcta. A pesar del inconveniente de realizar pronósticos de estas series, se procede a utilizar este modelo, ya que el mismo presenta mejor desempeño que la restante opción. Por último, la especificación seleccionada para la serie del ICC tiene como limitante que ningún rezago del IPPC es significativo. Sin embargo, sí se toma en cuenta el décimo rezago del IPPC $\left(I P P C_{t-10}\right)$, para mantener uniformidad en el análisis y para evaluar si la inclusión del IPPC aumenta la capacidad predictiva del modelo. El criterio de selección del rezago del IPPC consiste en, que a pesar de que ningún rezago es significativo, se eligió el que le aumentara la bondad de ajuste al modelo.

Los resultados de las pruebas de evaluación para los pronósticos de las series en estudios se muestran a continuación:

- Índice de Precios al Consumidor: con respecto a todas las pruebas aplicadas a esta serie, se concluye que el modelo ARDL tiene una mejor capacidad de pronóstico, ya que no se rechaza la hipótesis de insesgamiento del estimador. Además, mediante la prueba de Diebold y Mariano, se rechaza la hipótesis nula de igual poder predictivo de ambos modelos, en favor de mayor capacidad predictiva del ARDL. Por último, el ARDL posee una mejor capacidad de predicción en la dirección de los movimientos.

CUADRO 6

PRUEBAS DE CAPACIDAD DE PRONÓSTICO SOBRE LA SERIE DE VARIACIONES MENSUALES DEL ÍNDICE DE PRECIOS AL CONSUMIDOR

\begin{tabular}{lccc}
\hline Prueba & $\begin{array}{c}\text { Pronóstico } \\
\text { ARDL }\end{array}$ & $\begin{array}{c}\text { Pronóstico } \\
\text { ARMA }\end{array}$ & Mejor desempeño \\
\hline Insesgamiento $^{6}$ (p-value) & 0,07 & 0,00 & ARDL \\
Diebold-Mariano $^{7}$ (p-value) & 0,04 & 0,96 & ARDL \\
$\begin{array}{l}\text { Capacidad de predecir dirección de } \\
\text { movimiento }\end{array}$ & $66,7 \%$ & $55,6 \%$ & ARDL \\
RECM & 0,37 & 0,47 & ARDL \\
\hline
\end{tabular}

Fuente: Elaboración propia.

El insesgamiento se comprueba si no se rechaza la hipótesis conjunta $\alpha=0$ y $\beta=1$.

La prueba de Diebold y Mariano se le realiza a cada una de las series, en donde en ambos casos la hipótesis nula es de igual poder predictivo en cada par de series. La hipótesis alternativa en el caso de los pronóstico del modelo ARDL (segunda columna del cuadro) es que los pronósticos ARDL tienen mejor poder predictivo que los pronóstico ARMA. 
- Expectativas de inflación a 12 meses: para los pronósticos de ambos modelos no se rechaza la hipótesis de insesgamiento del pronóstico. La prueba de Diebold y Mariano sugiere que el ARDL tiene mayor poder predictivo. Además, el ARDL tiene una mayor capacidad de predecir la dirección de los movimientos. Se concluye que en este caso los pronósticos ARDL poseen una mejor capacidad predictiva que los ARMA.

CUADRO 7

PRUEBAS DE CAPACIDAD DE PRONÓSTICO SOBRE LA SERIE DE VARIACIONES INTERANUALES PARA LAS EXPECTATIVAS DE INFLACIÓN

\begin{tabular}{lccc}
\hline Prueba & $\begin{array}{c}\text { Pronóstico } \\
\text { ARDL }\end{array}$ & $\begin{array}{c}\text { Pronóstico } \\
\text { ARMA }\end{array}$ & Mejor desempeño \\
\hline Insesgamiento (p-value) & 0,88 & 0,06 & Igual \\
$\begin{array}{l}\text { Diebold-Mariano (p-value) } \\
\text { Capacidad de predecir dirección de }\end{array}$ & 0,05 & 0,95 & ADRL \\
movimiento & $61,1 \%$ & $50,0 \%$ & ARDL \\
RECM & 0,002 & 0,003 & ARDL \\
\hline
\end{tabular}

Fuente: Elaboración propia.

- Índice de confianza del consumidor: presenta características muy similares a los modelos de la serie anterior, ya que en ambos casos se rechaza la hipótesis de insesgamiento y no se rechaza la hipótesis nula de la prueba de Diebold y Mariano. Se diferencia en que sí tiene una mejor capacidad de predecir la dirección de los movimientos, la cual es mayor para los pronósticos del modelo ARMA. Asimismo, ambos modelos presentan una baja RECM, por lo que se concluye que los pronósticos ARMA tienen una mejor capacidad predictiva que los ARDL.

CUADRO 8

PRUEBAS DE CAPACIDAD DE PRONÓSTICO SOBRE LA SERIE DE

VARIACIONES TRIMESTRALES DEL ICC

\begin{tabular}{lccc}
\hline Prueba & $\begin{array}{c}\text { Pronóstico } \\
\text { ARDL }\end{array}$ & $\begin{array}{c}\text { Pronóstico } \\
\text { ARMA }\end{array}$ & Mejor desempeño \\
\hline $\begin{array}{l}\text { Insesgamiento (p-value) } \\
\text { Diebold-Mariano (p-value) }\end{array}$ & 0,00 & 0,00 & Igual \\
$\begin{array}{l}\text { Capacidad de predecir dirección de } \\
\text { movimiento }\end{array}$ & 0,64 & 0,36 & Igual \\
RECM & $50,0 \%$ & $66,7 \%$ & ARMA \\
\hline
\end{tabular}

Fuente: Elaboración propia. 
- IPC-estrato alto: en ambos modelos se rechaza la hipótesis de insesgamiento, pero tiene baja capacidad de predecir dirección de los movimientos. La prueba de Diebold y Mariano indica que el ARMA tiene mejor poder predictivo. De este modo, se concluye ARMA tienen una mejor capacidad de pronóstico.

CUADRO 9

PRUEBAS DE CAPACIDAD DE PRONÓSTICO SOBRE LA SERIE DE VARIACIONES MENSUALES DEL IPC-ESTRATO ALTO

\begin{tabular}{lccc}
\hline Prueba & $\begin{array}{c}\text { Pronóstico } \\
\text { ARDL }\end{array}$ & $\begin{array}{c}\text { Pronóstico } \\
\text { ARMA }\end{array}$ & Mejor desempeño \\
\hline $\begin{array}{l}\text { Insesgamiento (p-value) } \\
\text { Diebold-Mariano (p-value) }\end{array}$ & 0,00 & 0,00 & Igual \\
$\begin{array}{l}\text { Capacidad de predecir dirección de } \\
\text { movimiento }\end{array}$ & 0,96 & 0,04 & ARMA \\
RECM & $11,1 \%$ & $22,2 \%$ & ARMA \\
\hline
\end{tabular}

Fuente: Elaboración propia.

- IPC-estrato medio: en ambos modelos se rechaza la hipótesis de insesgamiento y no se rechaza la hipótesis nula de igual poder predictivo de la prueba de Diebold y Mariano. Además, presentan una baja capacidad de predecir dirección de los movimientos. Por lo tanto, para el IPC estrato medio, a pesar de las limitaciones que tienen ambos modelos, se concluye que no existe diferencia entre los modelos utilizados, ya que el criterio para concluir a favor de ARMA es el porcentaje de acierto y no llega al 50\% esperable.

CUADRO 10

PRUEBAS DE CAPACIDAD DE PRONÓSTICO SOBRE LA SERIE DE VARIACIONES MENSUALES DEL IPC-ESTRATO MEDIO

\begin{tabular}{lccc}
\hline Prueba & $\begin{array}{c}\text { Pronóstico } \\
\text { ARDL }\end{array}$ & $\begin{array}{c}\text { Pronóstico } \\
\text { ARMA }\end{array}$ & Mejor desempeño \\
\hline Insesgamiento (p-value) & 0,01 & 0,00 & Igual \\
Diebold-Mariano (p-value) & 0,23 & 0,77 & Igual \\
$\begin{array}{l}\text { Capacidad de predecir dirección de } \\
\text { movimiento }\end{array}$ & $33,3 \%$ & $38,9 \%$ & ARMA \\
RECM & 0,45 & 0,47 & ARDL \\
\hline
\end{tabular}

Fuente: Elaboración propia.

- IPC-estrato bajo: los pronósticos de los modelos de la serie IPC estrato bajo presentan menos limitantes que en las dos anteriores series, ya que para ambos modelos no se rechaza la hipótesis de insesgamiento del estimador, y en la prueba de Diebold y Mariano no se rechaza la hipótesis nula de igual poder predictivo. Dado que el ARDL tiene una capacidad de predecir la dirección de los movimientos considerablemente mayor que el ARMA, se concluye que en la serie del IPC estrato bajo los pronósticos ARDL tienen una mejor capacidad predictiva. 


\section{CUADRO 11}

PRUEBAS DE CAPACIDAD DE PRONÓSTICO SOBRE LA SERIE DE VARIACIONES MENSUALES DEL IPC-ESTRATO BAJO

\begin{tabular}{lccc}
\hline Prueba & $\begin{array}{c}\text { Pronóstico } \\
\text { ARDL }\end{array}$ & $\begin{array}{c}\text { Pronóstico } \\
\text { ARMA }\end{array}$ & Mejor desempeño \\
\hline Insesgamiento (p-value) & 0,15 & 0,69 & Igual \\
Diebold-Mariano (p-value) & 0,45 & 0,55 & Igual \\
Capacidad de predecir dirección de & & & \\
movimiento & $72,2 \%$ & $55,6 \%$ & ARDL \\
RECM & 0,31 & 0,31 & Igual \\
\hline
\end{tabular}

Fuente: Elaboración propia.

- IPC-transables: en el caso del pronóstico ARDL no se rechaza la hipótesis de insesgamiento del estimador, mientras que para el ARMA, sí. Además, en la prueba de Diebold y Mariano se rechaza la hipótesis nula de igual poder predictivo en ambos casos, se concluye que el modelo ARDL tiene mejor poder predictivo. Por último, el ARDL posee una mejor capacidad de predecir la dirección de los movimientos, por lo que para el IPC transables se deduce que los pronósticos ARDL tienen una mejor capacidad predictiva que los ARMA.

CUADRO 12

PRUEBAS DE CAPACIDAD DE PRONÓSTICO SOBRE LA SERIE DE VARIACIONES MENSUALES DEL IPC-TRANSABLES

\begin{tabular}{lccc}
\hline Prueba & $\begin{array}{c}\text { Pronóstico } \\
\text { ARDL }\end{array}$ & $\begin{array}{c}\text { Pronóstico } \\
\text { ARMA }\end{array}$ & Mejor desempeño \\
\hline Insesgamiento (p-value) & 0,41 & 0,03 & ARDL \\
Diebold-Mariano (p-value) & 0,07 & 0,93 & ARDL \\
$\begin{array}{l}\text { Capacidad de predecir dirección de } \\
\text { movimiento }\end{array}$ & $61,1 \%$ & $55,6 \%$ & ARDL \\
RECM & 0,62 & 0,76 & ARDL \\
\hline
\end{tabular}

Fuente: Elaboración propia.

- IPC-no transables: ambos modelos presentan limitaciones, ya que se rechaza la hipótesis de insesgamiento. Además, no se rechaza la hipótesis nula de igual poder predictivo de la prueba de Diebold y Mariano. Los dos modelos también muestran una baja capacidad de predecir la dirección de los movimientos. Por lo tanto, en el caso del IPC No Transables se concluye que no existe evidencia suficiente para determinar cuál modelo tiene una mejor capacidad predictiva. 


\section{CUADRO 13}

PRUEBAS DE CAPACIDAD DE PRONÓSTICO SOBRE LA SERIE DE

VARIACIONES MENSUALES DEL IPC-NO TRANSABLES

\begin{tabular}{lccc}
\hline Prueba & $\begin{array}{c}\text { Pronóstico } \\
\text { ARDL }\end{array}$ & $\begin{array}{c}\text { Pronóstico } \\
\text { ARMA }\end{array}$ & Mejor desempeño \\
\hline Insesgamiento (p-value) & 0,00 & 0,00 & Igual \\
Diebold-Mariano (p-value) & 0,27 & 0,73 & Igual \\
Capacidad de predecir dirección de & & & \\
movimiento & $33,3 \%$ & $38,9 \%$ & ARMA \\
RECM & 0,67 & 0,70 & ARDL \\
\hline
\end{tabular}

Fuente: Elaboración propia.

En resumen, los modelos que poseen una mejor capacidad predictiva para cada serie son los siguientes:

CUADRO 14

MODELOS CON MEJOR CAPACIDAD PREDICTIVA PARA CADA SERIE

\begin{tabular}{lc}
\hline Serie & Especificación \\
\hline IPC & ARDL \\
IPC-estrato alto & ARMA \\
IPC-estrato medio & - \\
IPC-estrato bajo & ARDL \\
IPC-bienes transables & ARDL \\
IPC-bienes no transables & - \\
Índice de Confianza del Consumidor (ICC) & ARMA \\
Expectativas de inflación a 12 meses & ARDL \\
\hline
\end{tabular}

Fuente: Elaboración propia.

Los resultados de la evaluación sugieren que el IPPC puede ser útil en la modelación del IPC de los estratos bajos, que es el grupo más expuesto y más perjudicado por los cambios en los precios. El IPPC también podría resultar útil para la modelación de las expectativas de inflación en Costa Rica, un tema relativamente poco desarrollado aparte de las contribuciones de Muñoz y Torres (2006) y más recientemente el de Alfaro y Monge (2013). 


\section{CONCLUSIONES Y RECOMENDACIONES}

Este artículo presentó el cálculo de un indicador de la percepción de la inflación por parte de los consumidores costarricenses, así como una evaluación de su capacidad predictiva, lo cual es un esfuerzo que no se había realizado anteriormente para Costa Rica.

En general, las variaciones interanuales del IPPC presentan una media $y$ variabilidad menores que el IPC por estratos sociales, $y$ las variantes del IPC por transables y no transables. El análisis del indicador muestra que productos consumidos con mayor frecuencia, como alimentos y gasolina, tienen mayor importancia en la determinación de la inflación percibida, tal como lo plantea Brachinger (2006, 2008). Los artículos comprados semanalmente aportan más del $90 \%$ de la media de la variación interanual mensual del IPPC, en comparación con 52\% en el caso del IPC.

En cuanto a la capacidad predictiva de ambos modelos, se puede concluir que los ARMA presentan mejores características para las variables IPC de estrato alto, IPC de estrato medio, IPC no transables e ICC; mientras tanto, los modelos ARDL tienen una mejor capacidad predictiva para las variables IPC, IPC de estrato bajo, IPC transables y expectativas de inflación. En este último caso, los modelos ARDL tienen un notable desempeño en el pronóstico, ya que cumplen con todos los criterios de evaluación. Se considera que el IPPC puede ser útil para modelar expectativas de inflación y las variaciones de los precios del estrato de ingresos bajos.

Una primera extensión del trabajo expuesto en este artículo es el cálculo del IPPC a partir del IPC con base junio del 2015, que incorpora mejoras conceptuales, metodológicas, así como una nueva canasta de 315 bienes y servicios. Como recomendación adicional, sería útil dar seguimiento mensual al cálculo del IPPC actualizado.

En esta investigación se utilizó modelos ARMA y ARDL por la simplicidad teórica y operacional. Sin embargo, la información del IPPC también podría también incorporarse en modelos de proyección más complejos, como VAR, SVAR o modelos semiestructurales. Asimismo, resultaría útil contrastar los resultados de este tipo de medidas basadas en postulados teóricos con información proveniente de encuestas, un esfuerzo que no se ha realizado todavía en Costa Rica.

\section{REFERENCIAS}

Alfaro, A., \& Monge, C. (2013). Expectativas de inflación para Costa Rica. (Documento de Investigación DI-01-2013), Departamento de Investigación Económica, BCCR. Recuperado de http://www.bccr.fi.cr/investigacioneseconomicas/politicamonetariaeinflacion/Expectativas_ inflacion_CR_por_estratos.pdf

Banco Central Europeo. (Mayo, 2007). Inflación observada e inflación percibida en la zona euro. Boletín Mensual, 5, 65-75.

Brachinger, H. W. (2006). Euro or "Teuro"?: The Euro-Induced Perceived Inflation in Germany. (Working Paper Series 5), Department of Quantitative Economics, University of Fribourg Switzerland.

Brachinger, H. W. (2008). A new index of perceived inflation: Assumptions, method, and application to Germany. Journal of economic psychology, 29(4), 433-457. http://dx.doi.org/10.1016/j.joep.2008.04.004

Diebold, F. \& Mariano, R. (1995). Comparing Predictive Accuracy. Journal of Business and Economic Statistics, 13, 253-265. http://dx.doi.org/10.1080/07350015.1995.10524599 
Fritsche, U., Vogel, L., \& Menz, J. O. (2009). Prospect Theory and Inflation Perceptions - An empirical assessment. (DEP Discussion Papers. Macroeconomics and Finance, Series 3). Department Economics and Politics, University of Hamburg.

Georganas, S., Healy, P. J., \& Li, N. (Abril, 2014). Frequency bias in consumers' perceptions of inflation: An experimental study. European Economic Review, 67, 144-158. http://dx.doi.org/10.1016/j.euroecorev.2014.01.014

Haschka, P. (2004). Verbraucherpreisindex - täglicher Einkauf und gefühlte. Inflation, Statistische Nachrichten, 3, 199-204.

Hendry, D. F. (1979). Predictive Failure and Econometric Modelling in Macroeconomics: The Transactions Demand for Money. Economic Modelling. 217-242. http://dx.doi.org/10.1093/0198293542.003.0012

Holden, K., \& Peel, D. (1989). Unbiasedness, Efficiency and the Combination of Economic Forecasts. Journal of Forecasting, 8, 175-188. http://dx.doi.org/10.1002/for.3980080304

INEC (2006). Encuesta Nacional de Ingresos y Gastos de los Hogares 2004: Metodología. San José: Instituto Nacional de Estadística y Censos (INEC).

Kahneman, D., \& Tversky, A. (1973). On the Psychology of Prediction. Psychological Review 80(4), 237-257. http://dx.doi.org/10.1037/h0034747

Kahneman, D. \& Tversky, A. (1981). The Framing of Decisions and the Psychology of Choice. Science, 211(4481), 453-458. http://dx.doi.org/10.1126/science.7455683

Kapounek, S., \& Lacina, L. (2011). Inflation Perception and Anticipation Gaps in the Eurozone.

(Working paper No. 4/2011), Faculty of Business and Economics, Mendel University in Brno.

Mata, E. (2013). Desempleo y alto costo de vida desvelan más a ticos [en línea]. La Nación. Recuperado de: http:/www.nacion.com/archivo/Desempleo-alto-costo-desvelan-ticos_0_1328867163.html

Muñoz, E. \& Torres, C. (2006). Un modelo de formación de expectativas de inflación para Costa Rica. (Documento de Investigación DEC-DIE-003-2006), Departamento de Investigación Económica, BCCR. Recuperado de: http://www.bccr.fi.cr/investigacioneseconomicas/politicamonetariaeinflacion/Modelo_formacion_expectativas_inflacion.pdf

Romina, L. (2007). Perceived Inflation and the Introduction of the Euro Currency in Malta. Bank of Valletta review, 36 .

Torres, C. (2012). Costa Rica: Determinación de cambios estructurales en el nivel de la tasa de inflación: periodo 1997-2011. (Documento de Trabajo 02-2012), Departamento de Investigación Económica, BCCR. Recuperado de: http://www.bccr.fi.cr/investigacioneseconomicas/politicamonetariaeinflacion/DT-02-2012_Determinacion_cambios_estructurales_inflacion1997-2011.pdf 


\section{ANEXOS}

Resultados de los pronósticos para cada serie:

GRÁFICO 4

PRONÓSTICOS DEL IPC

JULIO 2011 - DICIEMBRE 2012

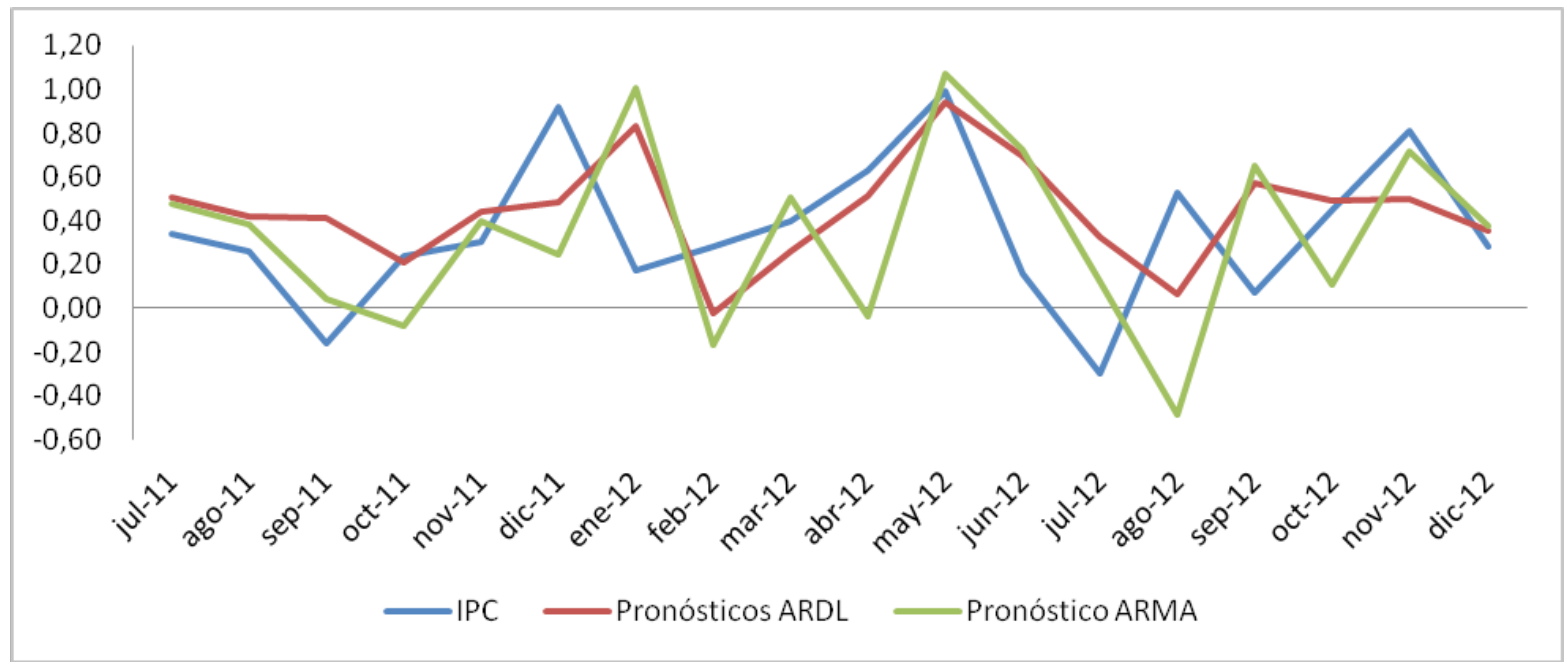

Fuente: Elaboración propia.

GRÁFICO 5

PRONÓSTICOS DEL IPC TRANSABLES

JULIO 2011 - DICIEMBRE 2012

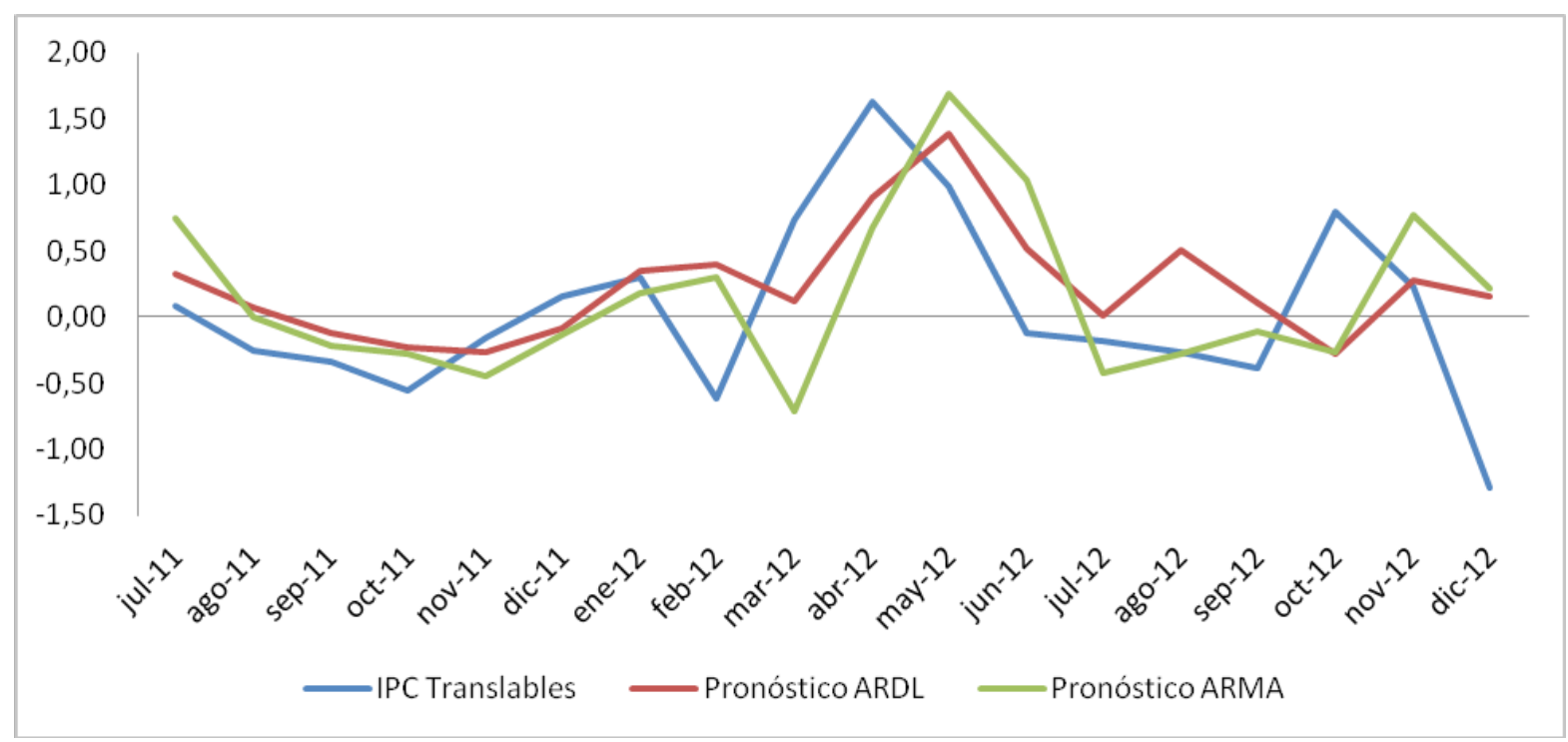

Fuente: Elaboración propia. 


\section{GRÁFICO 6}

PRONÓSTICOS DEL IPC NO TRANSABLES

JULIO 2011 - DICIEMBRE 2012

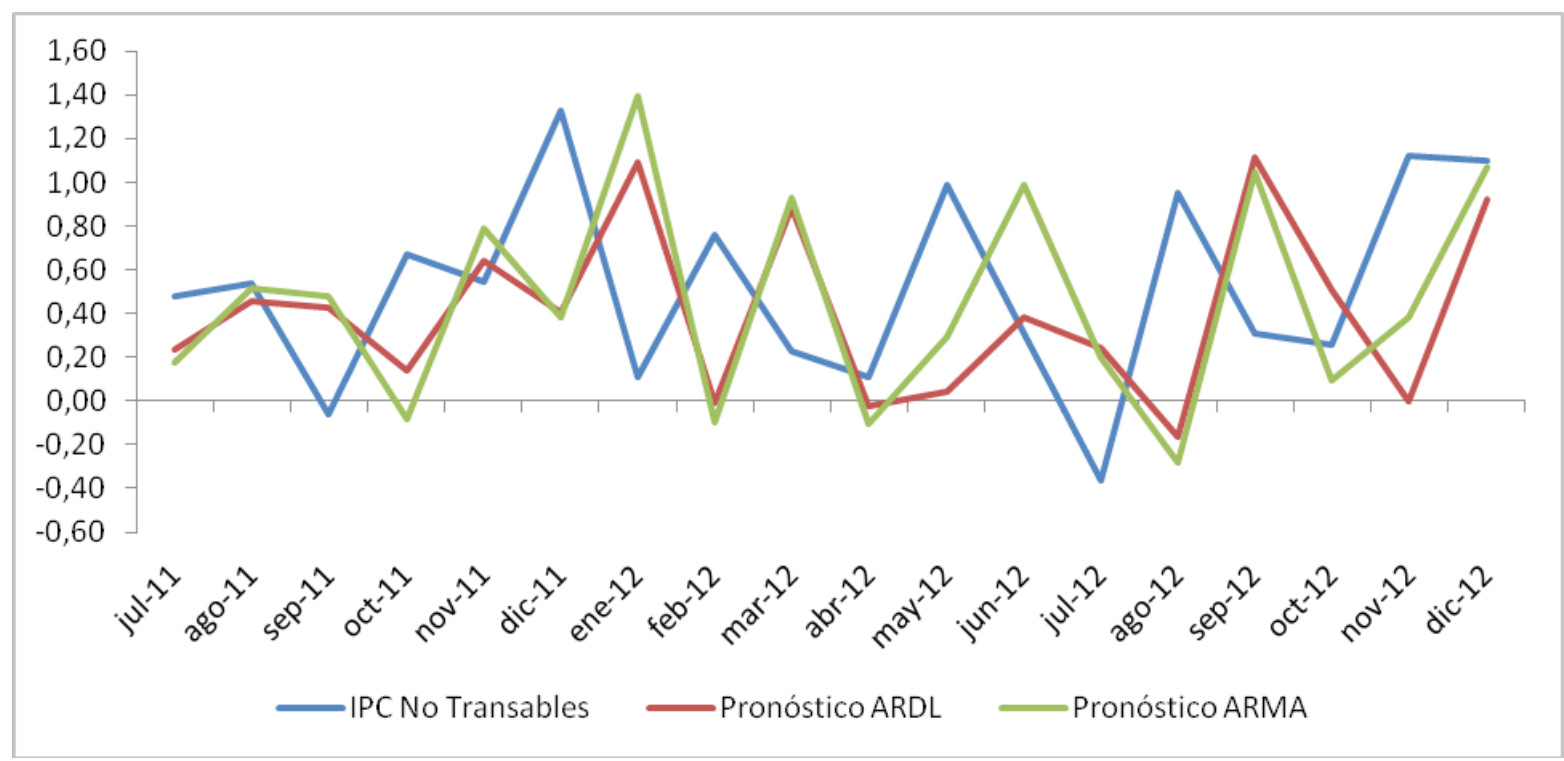

Fuente: Elaboración propia.

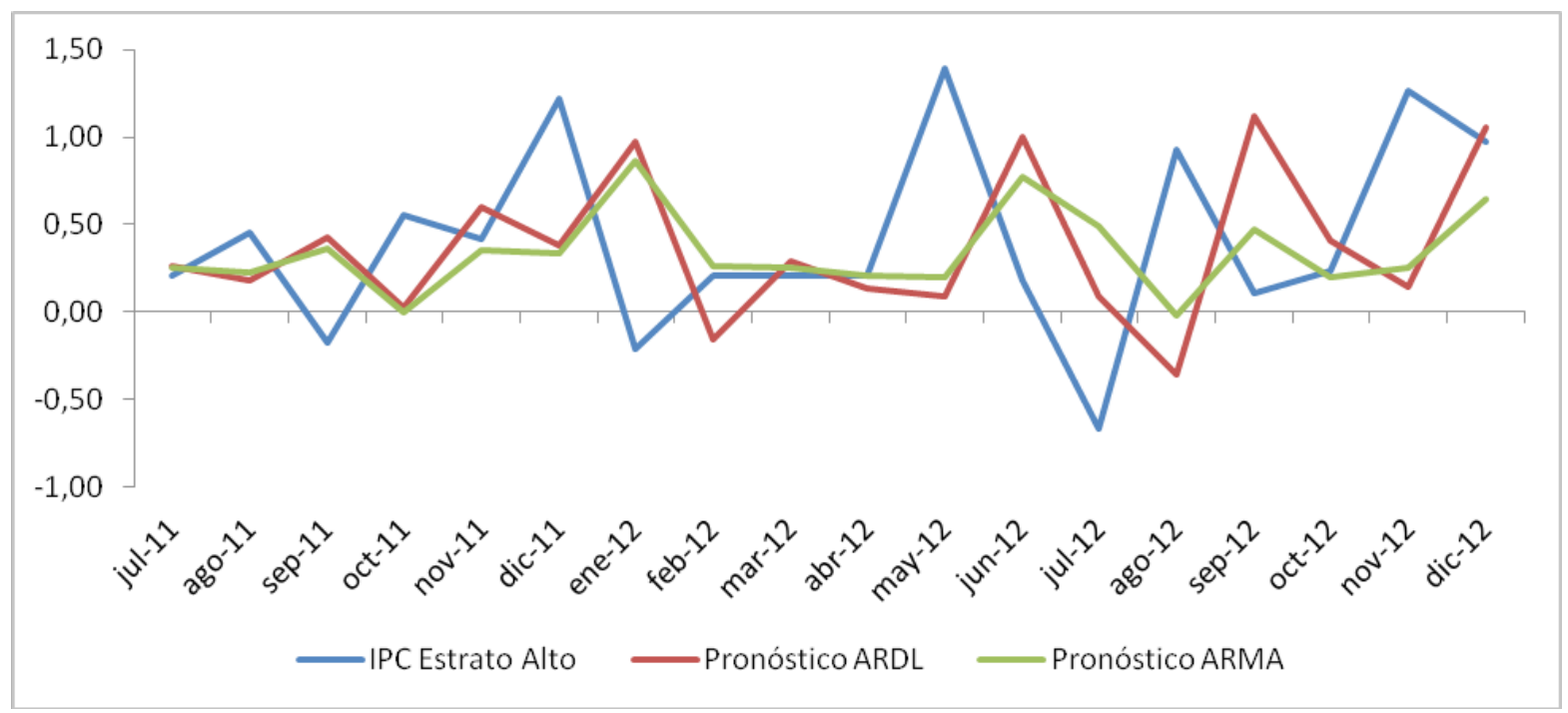

Fuente: Elaboración propia. 
GRÁFICO 8

PRONÓSTICOS DEL IPC ESTRATO MEDIO

JULIO 2011 - DICIEMBRE 2012

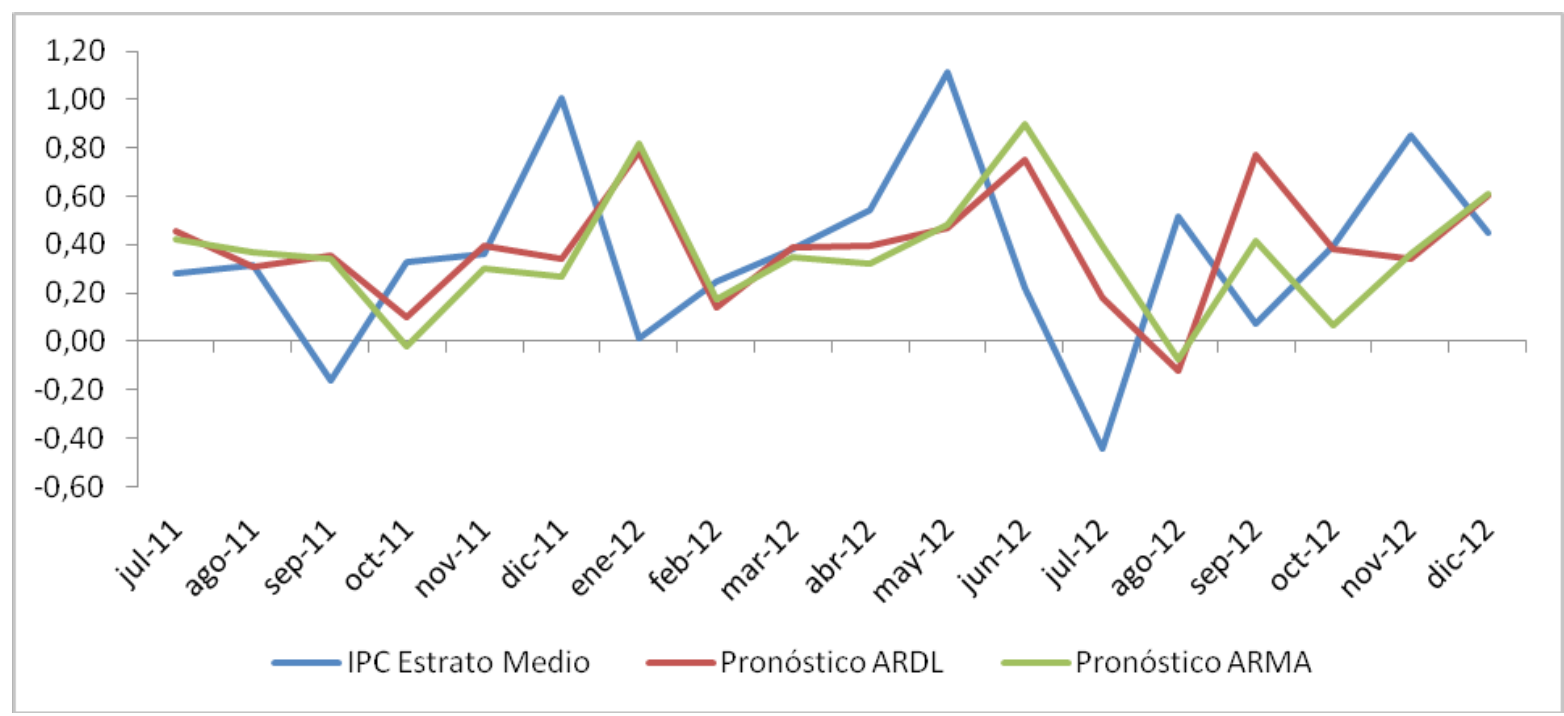

Fuente: Elaboración propia.

GRÁFICO 9

PRONÓSTICOS DEL IPC ESTRATO BAJO

JULIO 2011 - DICIEMBRE 2012

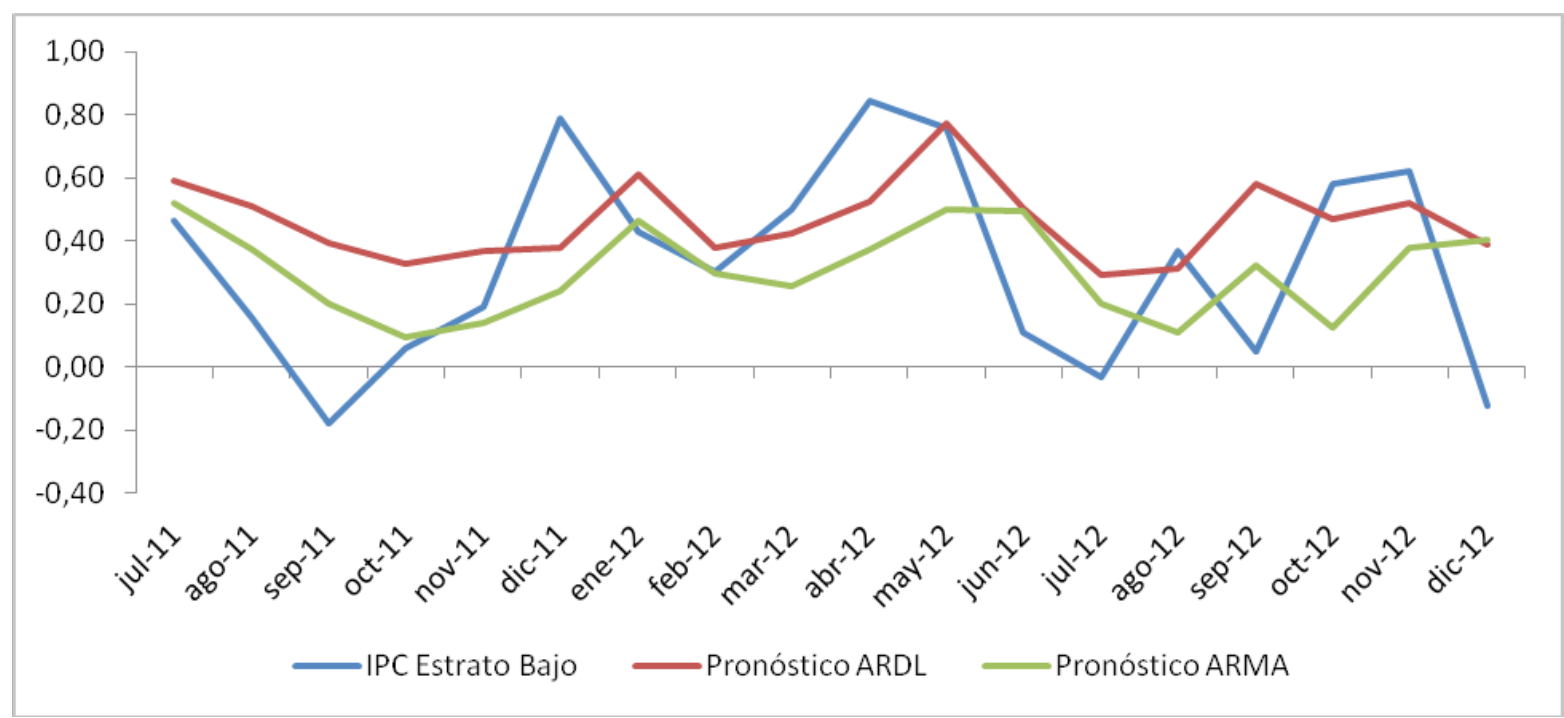

Fuente: Elaboración propia. 
GRÁFICO 10

PRONÓSTICOS DE LAS EXPECTATIVAS DE INFLACIÓN A 12 MESES

JULIO 2011 - DICIEMBRE 2012

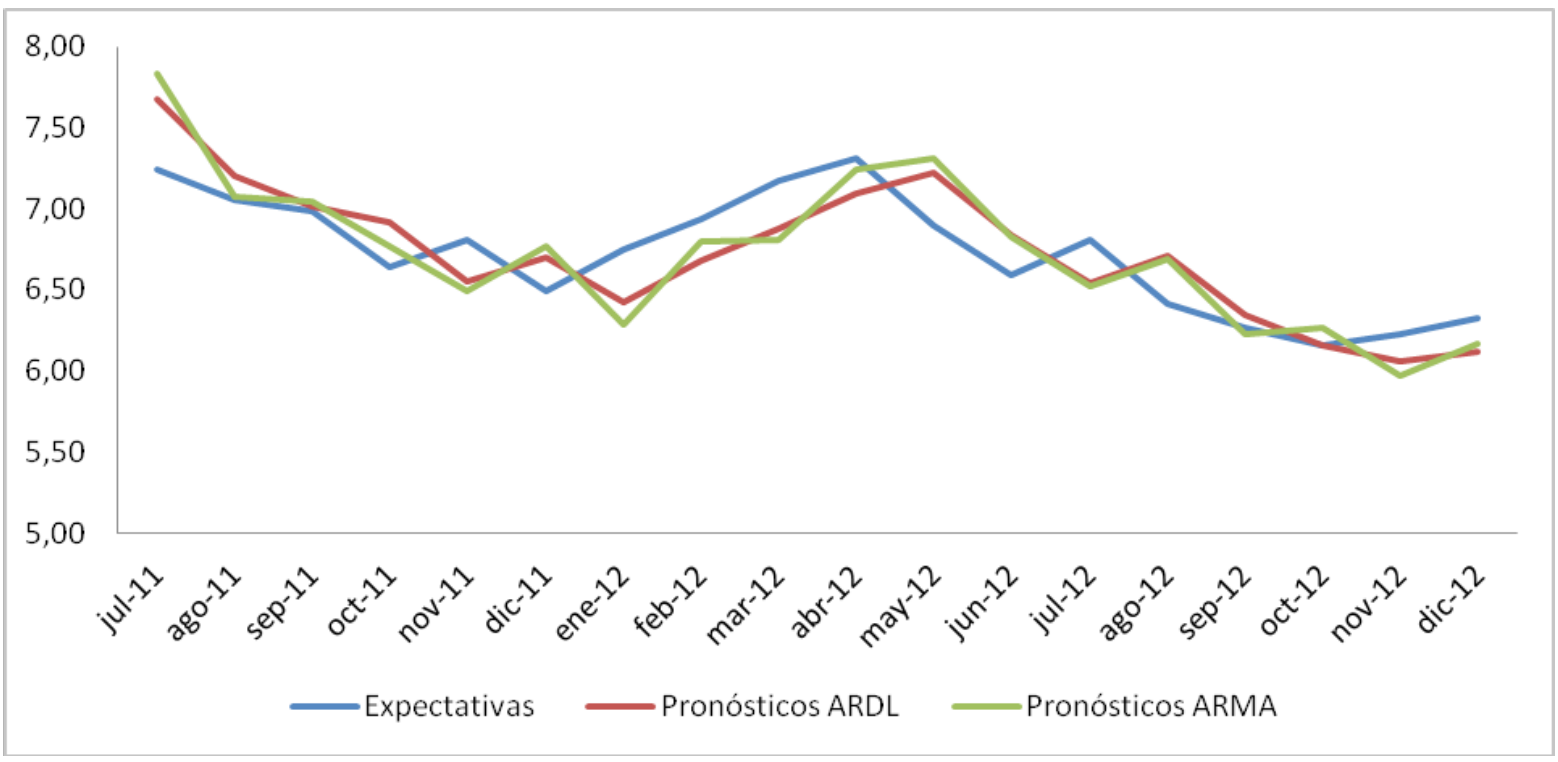

Fuente: Elaboración propia.

GRÁFICO 11

PRONÓSTICOS DEL ICC

T3-2011 - T4-2012

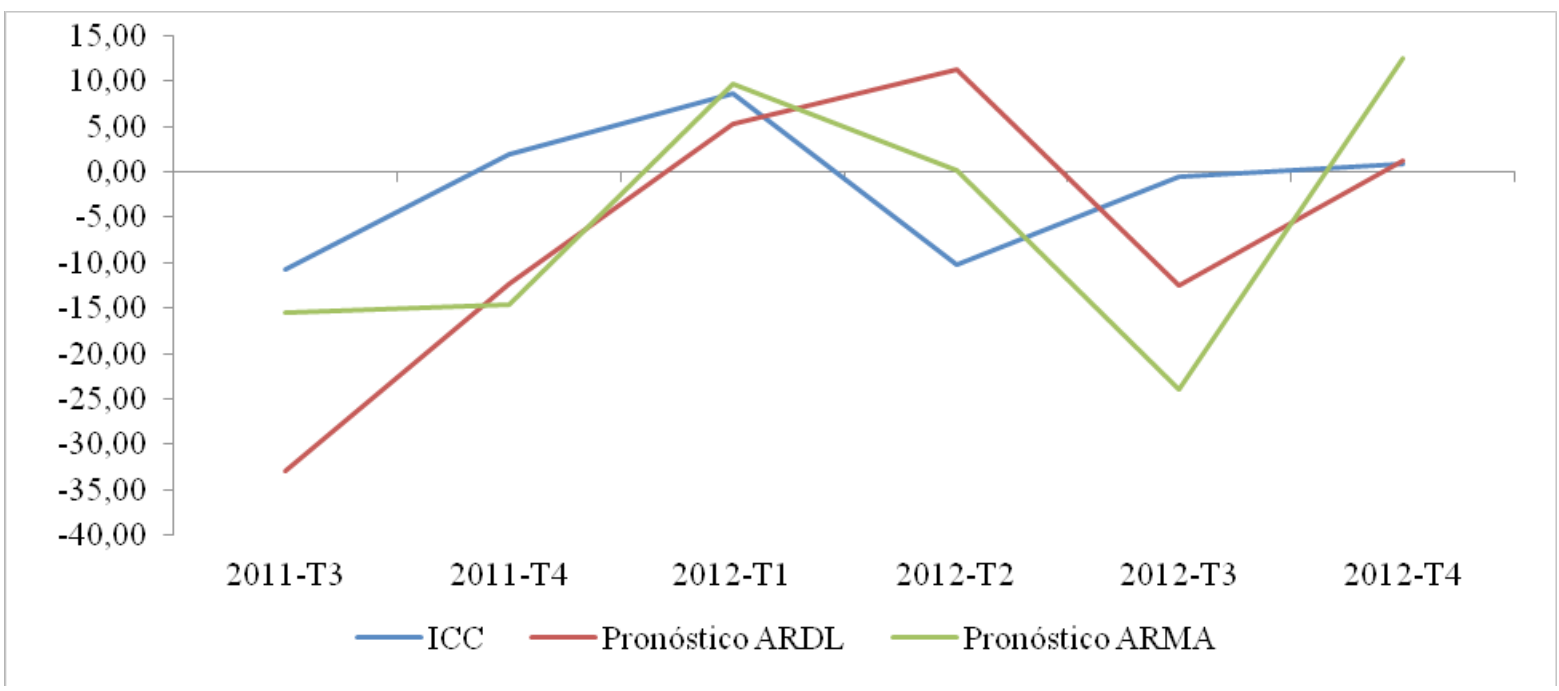

Fuente: Elaboración propia. 
Pruebas de verificación de diagnóstico

Pruebas de verificación de diagnóstico para las especificaciones ARMA seleccionadas para cada serie:

CUADRO 15

ESPECIFICACIÓN ARMA SELECCIONADA PARA LA SERIE DE VARIACIONES MENSUALES DEL ÍNDICE DE PRECIOS AL CONSUMIDOR

\begin{tabular}{ll}
\hline Criterio & Resultado \\
\hline Modelo seleccionado & ARMA $(2,4)$ \\
Significancia de Coeficientes $^{8}$ & $\operatorname{AR}(2)=(0,34)^{* *}$ MA $(4)=(-0,18)^{* *}$ \\
Bondad de Ajuste & $R^{2}=0,09$ \\
Normalidad de los errores & \\
Estacionariedad & Jarque-Bera $=4,84$ \\
\hline
\end{tabular}

Fuente: Elaboración propia.

CUADRO 16

ESPECIFICACIÓN ARMA SELECCIONADA PARA LA SERIE DE

VARIACIONES MENSUALES DEL IPC-ESTRATO ALTO

\begin{tabular}{ll}
\hline Criterio & Resultado \\
\hline Modelo seleccionado & $\operatorname{ARMA}(2,1)$ \\
Significancia de Coeficientes & $\operatorname{AR}(2)=(-0,18) \operatorname{MA}(1)=(0,69)^{* *}$ \\
Bondad de Ajuste & $R^{2}=0,46$ \\
Normalidad de los errores & Jarque-Bera $=1,45$ \\
Estacionariedad & El modelo es estacionario \\
\hline
\end{tabular}

Fuente: Elaboración propia.

$8 \quad *=$ Significativo al $1 \% * *=$ Significativo al $5 \% * * *=$ Significativo al $10 \%$.

$9 \quad$ El estadístico Jarque-Bera tiene una distribución Ji-cuadrado con 2 grados de libertad. Al 5\% de significancia el valor de este estadístico es de 5,99. La normalidad de los errores se comprueba con este estadístico y en esta prueba la hipótesis nula es la normalidad de los residuos por lo que se busca que no se rechace la hipótesis nula. 


\section{CUADRO 17}

ESPECIFICACIÓN ARMA SELECCIONADA PARA LA SERIE DE

VARIACIONES MENSUALES DEL IPC-ESTRATO MEDIO

\begin{tabular}{ll}
\hline Criterio & Resultado \\
\hline Modelo seleccionado & $\operatorname{ARMA}(1,2)$ \\
Significancia de Coeficientes & $\operatorname{AR}(1)=(0,47)^{* *} \operatorname{MA}(2)=(0,09)^{* *}$ \\
Bondad de Ajuste & $R^{2}=0,28$ \\
Normalidad de los errores & Jarque-Bera $=0,59$ \\
Estacionariedad & El modelo es estacionario \\
\hline
\end{tabular}

Fuente: Elaboración propia.

CUADRO 18

ESPECIFICACIÓN ARMA SELECCIONADA PARA LA SERIE DE

VARIACIONES MENSUALES DEL IPC-ESTRATO BAJO

\begin{tabular}{ll}
\hline Criterio & Resultado \\
\hline Modelo seleccionado & ARMA $(1,1)$ \\
Significancia de Coeficientes & $\operatorname{AR}(1)=(0,08) \operatorname{MA}(1)=(0,59) * *$ \\
Bondad de Ajuste & $R^{2}=0,50$ \\
Normalidad de los errores & Jarque-Bera $=5,48$ \\
Estacionariedad & El modelo es estacionario \\
\hline
\end{tabular}

Fuente: Elaboración propia.

CUADRO 19

ESPECIFICACIÓN ARMA SELECCIONADA PARA LA SERIE DE

VARIACIONES MENSUALES DEL IPC-BIENES TRANSABLES

\begin{tabular}{ll}
\hline Criterio & Resultado \\
\hline Modelo seleccionado & $\operatorname{ARMA}(3,4)$ \\
Significancia de Coeficientes & $\operatorname{AR}(3)=(0,03) \mathrm{MA}(4)=(-0,82)^{* *}$ \\
Bondad de Ajuste & $R^{2}=0,72$ \\
Normalidad de los errores & Jarque-Bera $=3,77$ \\
Estacionariedad & El modelo es estacionario \\
\hline
\end{tabular}

Fuente: Elaboración propia. 
CUADRO 20

ESPECIFICACIÓN ARMA SELECCIONADA PARA LA SERIE DE VARIACIONES MENSUALES DEL IPC-BIENES NO TRANSABLES

\begin{tabular}{ll}
\hline Criterio & Resultado \\
\hline Modelo seleccionado & $\operatorname{ARMA}(2,2)$ \\
Significancia de Coeficientes & $\operatorname{AR}(2)=(-0,97)^{* *} \operatorname{MA}(2)=(0,98)^{* * *}$ \\
Bondad de Ajuste & $R^{2}=0,37$ \\
Normalidad de los errores & Jarque-Bera $=2,18$ \\
Estacionariedad & El modelo es estacionario \\
\hline
\end{tabular}

Fuente: Elaboración propia.

CUADRO 21

ESPECIFICACIÓN ARMA SELECCIONADA PARA LA SERIE DE

VARIACIONES TRIMESTRALES DEL ICC

\begin{tabular}{ll}
\hline Criterio & Resultado \\
\hline Modelo seleccionado & $\operatorname{ARMA}(4,3)$ \\
Significancia de Coeficientes & $\operatorname{AR}(4)=(-0,56) * * \mathrm{MA}(3)=(0,13)$ \\
Bondad de Ajuste & $R^{2}=0,95$ \\
Normalidad de los errores & Jarque-Bera $=1,51$ \\
Estacionariedad & El modelo es estacionario. \\
\hline
\end{tabular}

Fuente: Elaboración propia.

CUADRO 22

ESPECIFICACIÓN ARMA SELECCIONADA PARA LA SERIE DE

VARIACIONES INTERANUALES PARA LAS EXPECTATIVAS DE INFLACIÓN

\begin{tabular}{ll}
\hline Criterio & Resultado \\
\hline Modelo seleccionado & $\operatorname{ARMA}(3,1)$ \\
Significancia de Coeficientes & $\operatorname{AR}(3)=(-0,30)^{* *} \operatorname{MA}(1)=(0,13)$ \\
Bondad de Ajuste & $R^{2}=0,98$ \\
Normalidad de los errores & Jarque-Bera $=5,22$ \\
Estacionariedad & El modelo es estacionario. \\
\hline
\end{tabular}

Fuente: Elaboración propia 
Pruebas de verificación de diagnóstico para las especificaciones ARDL seleccionadas para cada serie:

CUADRO 23

ESPECIFICACIÓN ARDL SELECCIONADA PARA LA SERIE DE VARIACIONES MENSUALES DEL ÍNDICE DE PRECIOS AL CONSUMIDOR

\begin{tabular}{ll}
\hline Criterio & Resultado \\
\hline Modelo seleccionado & $I P C_{t-1} I P P C_{t-1} I P P C_{t-5}$ \\
Significancia de Coeficientes & $I P C_{t-1}=(0,03) I P P C_{t-1}=(0,18)^{* * *}$ \\
Bondad de Ajuste & $I P P C_{t-5}=(0,30)^{* * *}$ \\
Normalidad de los errores & $R^{2}=0,34$ \\
Estacionariedad & Jarque-Bera $=6,16$ \\
\hline
\end{tabular}

Fuente: Elaboración propia.

CUADRO 24

ESPECIFICACIÓN ARDL SELECCIONADA PARA LA SERIE DE

VARIACIONES MENSUALES DEL IPC-ESTRATO ALTO

\begin{tabular}{ll}
\hline Criterio & Resultado \\
\hline Modelo seleccionado & $I P C_{-} E A_{t-12} I P P C_{t-1} I P P C_{t-5}$ \\
Significancia de Coeficientes & $I P C_{-} E A_{t-12}=(0,20)^{* *} I P P C_{t-1}=(0,38)^{* *}$ \\
Bondad de Ajuste & $I P C_{t-5}=(0,32)^{* *}$ \\
Normalidad de los errores & $R^{2}=0,41$ \\
Estacionariedad & Jarque-Bera $=1,02$ \\
\hline
\end{tabular}

Fuente: Elaboración propia.

CUADRO 25

ESPECIFICACIÓN ARDL SELECCIONADA PARA LA SERIE DE VARIACIONES MENSUALES DEL IPC-ESTRATO MEDIO

\begin{tabular}{ll}
\hline Criterio & Resultado \\
\hline Modelo seleccionado & $I P P C_{t-1} I P P C_{t-5}$ \\
Significancia de Coeficientes & $I P P C_{t-1}=(0,33)^{* * *} I P P C_{t-5}=(0,20)^{* *}$ \\
Bondad de Ajuste & $R^{2}=0,40$ \\
Normalidad de los errores & Jarque-Bera $=1,42$ \\
Estacionariedad & - \\
\hline
\end{tabular}


Fuente: Elaboración propia.

CUADRO 26

ESPECIFICACIÓN ARDL SELECCIONADA PARA LA SERIE DE

VARIACIONES MENSUALES DEL IPC-ESTRATO BAJO

\begin{tabular}{ll}
\hline Criterio & Resultado \\
\hline Modelo seleccionado & $I P C_{-} E B_{t-1} I P P C_{t-5}$ \\
Significancia de Coeficientes & $I P C_{-} E B_{t-1}=(0,37)^{* *} I P P C_{t-5}=(0,15)^{* * * *}$ \\
Bondad de Ajuste & $R^{2}=0,19$ \\
Normalidad de los errores & Jarque-Bera $=20,78$ \\
Estacionariedad & El modelo es estacionario \\
\hline
\end{tabular}

Fuente: Elaboración propia.

CUADRO 27

ESPECIFICACIÓN ARDL SELECCIONADA PARA LA SERIE DE

VARIACIONES MENSUALES DEL IPC-BIENES TRANSABLES

\begin{tabular}{ll}
\hline Criterio & Resultado \\
\hline Modelo seleccionado & $I P C_{-} T_{t-1} I P C_{-} T_{t-6} I P P C_{t-5}$ \\
Significancia de Coeficientes & $I P C_{-} T_{t-1}=(0,46)^{* *} I P C_{-} T_{t-6}=(-0,39)^{* * *}$ \\
Bondad de Ajuste & $I P P C_{t-5}=(0,29)^{* *}$ \\
Normalidad de los errores & $R^{2}=0,36$ \\
Estacionariedad & Jarque-Bera $=9,35$ \\
\hline
\end{tabular}

Fuente: Elaboración propia.

CUADRO 28

ESPECIFICACIÓN ARDL SELECCIONADA PARA LA SERIE DE

VARIACIONES MENSUALES DEL IPC-BIENES NO TRANSABLES

\begin{tabular}{ll}
\hline Criterio & Resultado \\
\hline Modelo seleccionado & $I P C_{-} N T_{t-12} I P P C_{t-1} I P P C_{t-3} I P P C_{t-5} I P P C_{t-6}$ \\
Significancia de Coeficientes & $I P C_{-} T_{t-1}=(0,46)^{* * *} I P C_{-} T_{t-6}=(-0,39)^{* *}$ \\
Bondad de Ajuste & $I P P C_{t-5}=(0,29)^{* * *}$ \\
Normalidad de los errores & $R^{2}=0,47$ \\
Estacionariedad & Jarque-Bera $=0,94$ \\
\hline
\end{tabular}

Fuente: Elaboración propia. 
CUADRO 29

ESPECIFICACIÓN ARDL SELECCIONADA PARA LA SERIE DE

VARIACIONES TRIMESTRALES DEL ICC

\begin{tabular}{ll}
\hline Criterio & Resultado \\
\hline Modelo seleccionado & $\mathrm{ICC}_{\mathrm{t}-6} \mathrm{IPPC}_{\mathrm{t}-10}$ \\
Significancia de Coeficientes & $\mathrm{ICC}_{\mathrm{t}-6}=(-0,57) * * \mathrm{IPPC}_{\mathrm{t}-10}=(0,82)$ \\
Bondad de Ajuste & $R^{2}=0,61$ \\
Normalidad de los errores & Jarque-Bera $=0,70$ \\
Estacionariedad & El modelo es estacionario. \\
\hline
\end{tabular}

Fuente: Elaboración propia.

CUADRO 30

ESPECIFICACIÓN ARDL SELECCIONADA PARA LA SERIE DE

VARIACIONES INTERANUALES PARA LAS EXPECTATIVAS DE INFLACIÓN

\begin{tabular}{ll}
\hline Criterio & Resultado \\
\hline Modelo seleccionado & $\operatorname{Exp}_{t-1}^{12} \operatorname{Exp}_{t-9}^{12} I P P C_{t-1} I P P C_{t-9} I P P C_{t-11}$ \\
Significancia de Coeficientes & $\operatorname{Exp}_{t-1}^{12}=(0,50)^{* *} \operatorname{Exp}_{t-9}^{12}=(0,23)^{* *}$ \\
Bondad de Ajuste & $R^{2}=0,99$ \\
Normalidad de los errores & Jarque-Bera $=0,34$ \\
Estacionariedad & El modelo es estacionario. \\
\hline
\end{tabular}

Fuente: Elaboración propia. 